Historic, Archive Document

Do not assume content reflects current scientific knowledge, policies, or practices. 







\section{Shipping Instructions}

SHIPMENTS, Freight, Express and Parcel Post. All retail orders of Peonies and Irises will be sent prepaid from coast to coast. No charges for packing.

WHOLESALE SHIPMENTS. Transportation charges collect. No charges for packing.

\section{SIZE OF PEONY PLANTS}

Prices quoted are forStandard 3 to 5 eye divisions or plants, with well balanced root systems. The size universally accepted as giving the most satisfactory results.

\section{SIZE OF IRIS RHIZOMES}

Strong, healthy, well grown single rhizoms.

\section{CUARANTEE}

All varieties are guaranteed to be true to name and in healthy condition, but should a mistake occur, we will gladly replace same.

Peonies and Irises are exceptionally hardy plants and seldom fail the most novice amateur, but we can assume no responsibility after they have passed beyond our control.

\section{PRICES}

Net cash. Cash with order unless credit rating can be shown or references given.

\section{SUBSTITUTIONS}

Are never made unless so requested and money will be promptly returned.

\section{REFERENCES}

Peoples Savings Bank and Van Wert National Bank, Van Wert, Ohio.

\section{EXHIBITION GARDENS}

C. F.Wassenberg gardens are located six squares from the centre of the city, within easy walking distance and in the heart of the Peony and Iris Garden district. Visitors always welcome.

C. F. WASSENBERG PEONY AND IRIS FARMS are located $4 \frac{1}{2}$ miles east, on Lincoln Highway.

\section{SHIPPING DATES}

Peonies-September 1st, until ground freezes.

Iris-July 1st, until ground freezes.

TO THE TRADE

Special quantity prices quoted on request. 


\section{DISCOUNTS}

Quantity discount is only allowed on retail orders accompanied with remittance in full, in advance, but in no case on collections.

Orders amounting to $\$ 25.00-10$ per cent.

Orders amounting to $\$ 50.00-15$ per cent.

Orders amounting to $\$ 100.00-20$ per cent.

\section{EARLY ORDERS}

Orders should, when possible, be placed early to insure securing the desired varieties and quantities.

\section{RATING OF VARIETIES}

The figures before the varieties give the ratings based on 100 per cent and were taken by members of both Peony and Iris Societies. This is of great assistance in selecting the most meritorious varieties. Those without ratings are new and have never been rated.

\section{NO DESCRIPTION EQUALS SEEING}

the varieties in the Garden or Show Table. Lovers of the Peony and Iris should avail themselves of every opportunity to see and enjoy the different varieties as many times during the season as possible. The Wassenberg Gardens, as well as our larger planting, the C. F. Wassenberg Peony and Iris Farms located $4 \frac{1}{2}$ miles east of the city on the Lincoln Highway are open to visitors and will be glad to welcome you at any time.

\section{PLAN TO SHOW}

Your Peonies and Iris at your own local Exhibition and, when possible, at the National Show. The joy of competition and being a part of the Show will more than repay your efforts, besides the pleasure given to thousands who do not possess a garden.

\section{MEMBER OF}

American Peony Society.

American Iris Society.

Commercial Peony and Iris Growers Association.

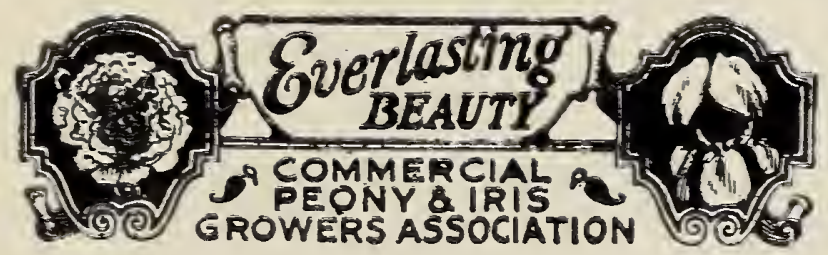




\section{INSTRUCTIONS FOR PLANTING PEONIES}

Peonies should be planted in good, average garden soil. For permanent planting, prepare the ground by lifting out the top soil-the depth of a spade-and mixing into the lower soil plenty of well rotted manure, being careful not to allow this to touch the peony roots. Replace top soil and plant peonies three to four feet apart, covering the eyes with about two inches of fine dirt, not more. Deeper planting will keep them from blooming and make weak, slow growing plants.

If manure is not available, loosen dirt as described above and place about a handful of bonemeal or wood ashes around each plant. After the ground freezes cover with two or three inches of leaves, straw, or other litter to protect against spring freezing. and thawing. Remove about April first. This is necessary only the first year.

Occasional cultivating from May to September will produce extra growth in the roots and give profuse bloom the following season.

Peonies should have plenty of sunshine and should not be too near old trees or shrubbery.

Never plant a peony where one has been planted before unless all the soil has been removed and replaced with soil that has never grown peonies, otherwise the plants will remain small and fail to bloom.

Before the blooming season, if the soil is dry, several liberal waterings at the base of the stems will increase the size of the blooms. For large show blooms cut off the small side buds as soon as large enough to see, this throws all the strength in the one flower and greatly increases its size.

For you who are planting peonies for the first time and do not know the wonderous beauty of the Modern Peony-many, many are the thrills that are in store for you. The peony. is without doubt the grandest of all perennials, with all the delicacy of the rose, only multiplied by a size that overwhelms. That is why people by the thousands come hundreds of miles to see the various Peony Farms and Gardens. No flower you can mention draws the thousands of visitors to a planting as do Peonies, and there is a reason.

Plan to visit a garden this year and see for yourself just how beautiful peonies are. 


\section{IRIS PLANTING INSTRUCTIONS}

Plant in good average garden soil to which may be added a little bone meal and some crushed limestone as Irises like lime. They do best when planted where they will receive plenty of sunshine, but they will also flower in partial shade though not as freely.

See that the place you expect to plant them is well drained so they will not be where they will stand in water, but where all water will run off quickly. Some Iris lovers make their beds six inches higher than the garden path.

Irises should be spaced 12 to 15 inches apart. They should be divided and replanted about every four years.

Place the roots two or three inches under the ground with about an inch of soil over the rhizome where the roots start.

The size of rhizomes vary greatly with the different varieties-some being large and fleshy while others are quite small. All are smaller in July and August than later-but we recommend planting in these months for best results. However, it is better to plant late than to wait another year.

Irises will come to you packed dry and should be planted as soon as received. Keep the plants free from weeds by frequent but shallow cultivating and far enough away from the plants so that their strength will not be taken by older or larger plants.

Beardless Irises will come to you with their roots kept moist. Do not let them dry out before planting as they are moisture loving plants, while the Bearded Irises are not.

Plant for massed effect to get the greatest beauty, where you can view in the early morning as the sun is coming up and the late afternoon as the sun is setting. You can then see them in all their artistic coloring from rich dark hues of purples and blues through smoky cloud-like shades of buff and grey to the delicate orchid tints and rainbow effects.

Of all the garden flowers of today the Iris has developed most rapidly and is now considered the most popular perennial in the United States. 


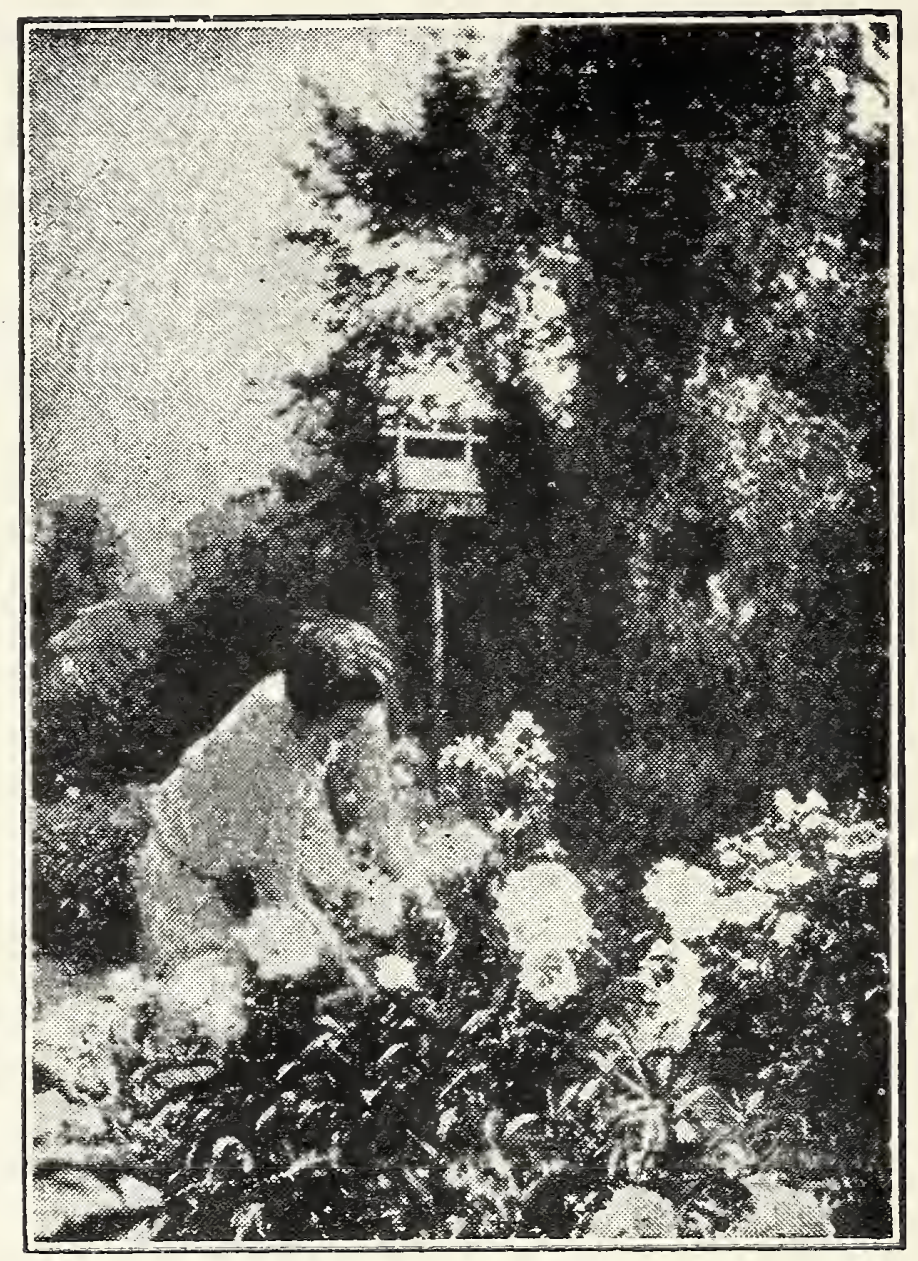

CUT BOUQUETS FOR YOUR HOME

Then more than ever will you marvel at their beauty

\section{PEONIES}

7.7 Adelaide Hollis ( $\mathrm{Hol}$ ) Lilac white

8.8 Adelanchea (Bra) Creamy white

each

$\$ 2.50$

7.1 Admiral Togo (Hol) Dark crimson ..... 1.50

8.5 Adolph Rosseau (Des) Deep garnet ...... 1.50

7.2 Aksarben (Ros) Brilliant dark crimson .... 1.00

8.7 Albatre (Cr) White. Large, fragrant . . . . . 1.00

8.6 Albert Crousse (Cr) Salmon pink ....... 1.00 Alice Balfour (Kel) Delicate rose pink ... 3.00

9.5 Alice Harding (Lem) Cream white ... . 75.00 Alpheus Hyatt (Rich) Rose pink. Late . . . 3.00

8.8 Alsace Lorraine (Lem) Cream white . . . . 2.00

8.6 A. P. Saunders (Thur) Flesh white ..... 10.00

7.5 Archie Brand (Bra) Shell pink ........ 1.50 
8.1 Arlequin (Des) Purpleish pink

each

8.1 Asa Gray (Cr) Pale lilac dotted

3.00

8.7 Auguste Dessert (Des) Crimson carmine

1.00

7.3 Auguste Villaume (Cr) Rose, late

7.50

8.0 Aurore (Des) Lilac white . . . . . . . . . . . 1.0

8.7 Avalanche (Cr) Cream white ....... 1.00

8.0 Aviator Reymond (Des) Cherry red .... 2.00

8.8 Ball O' Cotton (Franklin) Pure white ... 7.50

9.0 Baroness Schroeder (Kel) Flesh white. Good 1.50

9.0 Bayadere (Lem) Cream white .......... 3.50

Bay State Red ................... 1.50

8.0 Beau Marchais (Lem) Late pink ......... 7.50

8.9 Betty Blossom (Thur) Soft yellow effect . .20.00

7.9 Beauty's Mask (Hol) Blush white ....... 1.50 Benoit Dark red ................. . 3.00

8.6 Bertrade (Lem) Late white ........... 3.00 Blanch King (Bra) Deep pink . . . . . . . 50.00

8.7 Brand's Magnificent (Bra) Deep red ..... 5.00

8.4 Candeur (Des) Silvery pink .......... 3.00 Catherine (Rich) Pink ............ 3.00

Cecelia Kelway (Kel) Mauve pink ....... 1.50

8.4 Charlot (Doriat) Silvery lilac ...... . . 10.00

7.8 Chas McKellip (Bra) Rich red.......... 2.50

8.5 Chas Neidel (Wett) Blush pink ........ 2.50

8.7 Cherry Hill (Thur) Deep garnet ....... 3.50

8.4 Chestine Gowdy (Bra) Shell pink ....... 1.50

7.3 Christine Ritcher ( $\mathrm{Hol}$ ) Violet rose ....... 2.00

8.6 Clair Dubois $(\mathrm{Cr})$ Satiny pink .......... 1.00

Clara Anderson (And-Hol) White ...... 5.00

8.5 Clemenceau (Des) Carmine silver edge . . 5.00 Coronation (Kel) Pink, white center .... 3.00

9.1 Cornelia Shaylor (Shay) Faint pink. Late. . 12.50

7.9 C. S. Minot Pale lilac rose . . . . . . . . . 2.00

8.1 Couronne D' Or (Cal) Milk white ....... 1.00

David (Kel) Coral pink ............ . 3.50

8.4 David Harum (Bra) Bright red. Good .... 5.00

8.5 Desire (Bra) Delicate manve . .... 10.00

Directeur Ursat (Dor' 25) Flesh pink .... . 15.00

7.7 Dorchester (Rich) Hydrangea pink ... 1.00

9.1 Doris Shaylor (Shay) Fine pink ....... 5.00

Dr. Edgar Pleas (Pleas) Lilac pink ... 1.50

8.1 Dr. H. Barnsby (Des) Deep crimsnn .... 2.00

Duchess of Albany (Kel) Deep pink ... 3.00

Q.1 Duchesse de Nemours (Cal) White . 1.00

9.1 E. C. Shaw (Thur) Clear rose . . . . . . . 20.00

Edith Cavel (Kel) Milk white ........ 3.00

7.6 Edulis Superba (Lem) Dark pink .... 1.00

PEONIES- 3 to 5 eye divisions 


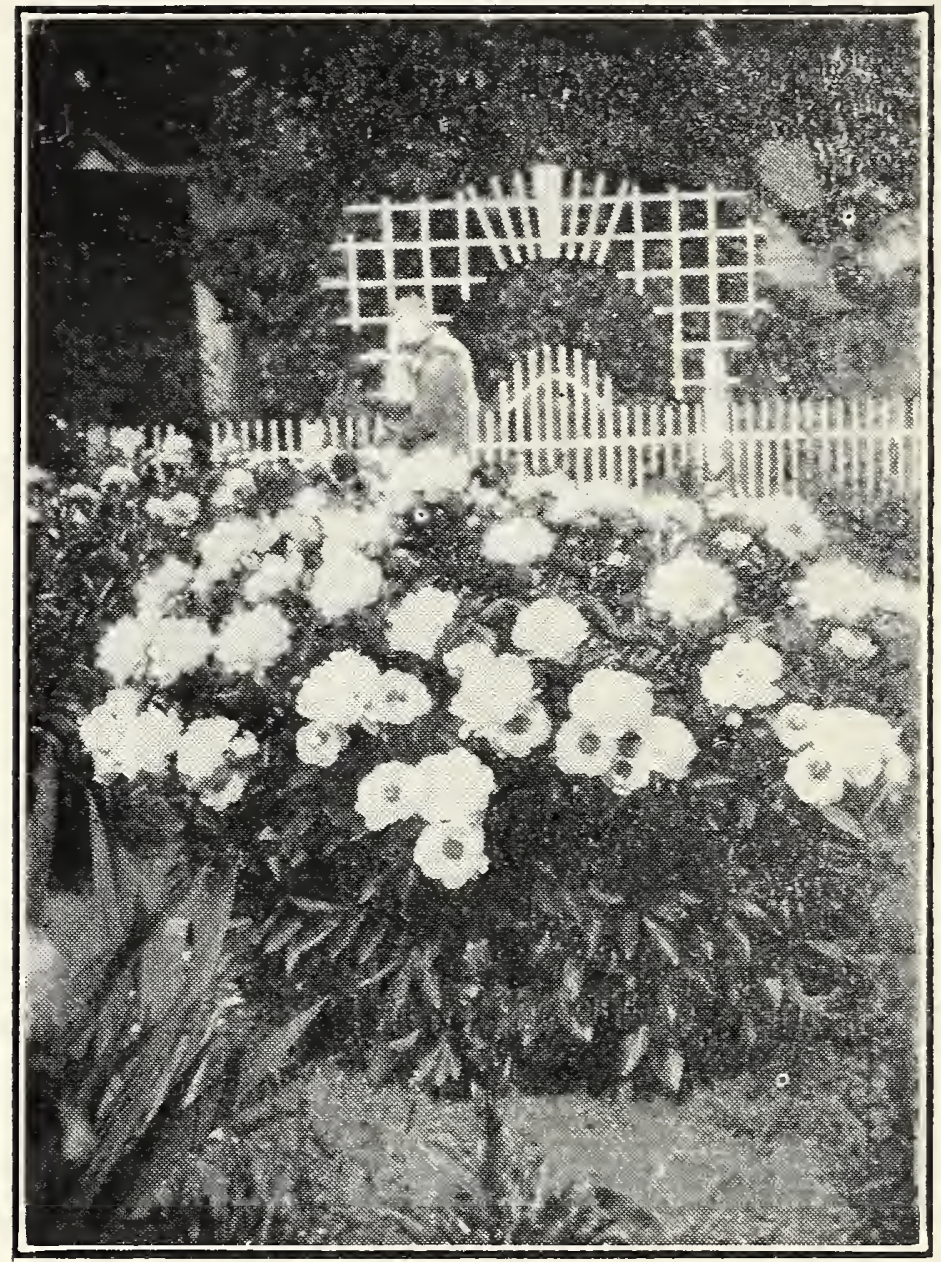

\section{PEONY MARIE JACQUIN}

Who would not be proud to have this plant in their garden?

(7.5each

7.5 Edwin Forrest (Hol) Dark Crimson ..... 2.00

8.7 E. J. Shaylor (Shay) Rose pink ........ 10.00

8.5 Elisa (Des) Hydrangea pink ... . . 10.00

9.1 Elizabeth B. Browring (Bra) Pure white.. 3.50

8.7 Elwood Pleas (Pleas) Light rose pink .... 2.50

8.9 Enchantress (Lem) Cream white ....... 3.50

7.9 Estafette (Des) Crimson, silvery margin . 2.50

8.0 Etta (Ter) Deep shell pink ........ 1.50

7.6 Eucharis (Lem) rreamy white ...... 2.00

8.3 Eugene Verdier (Cal) Pale hydrangea pink 1.50

8.6 Eugenie Verdier (Cal) Hydrangea pink ... 1.00

8.9 Eunice Shaylor (Shay) Ivory white ..... 6.00

8.3 Evening Glow ( $\mathrm{Hol})$ Lilac; late ........ 2.50

8.5 Exquisite (Kel) Rosy pink .......... 2.50

Fairvette Streaked crimson ….... 1.50

8.4 Felix Crousse (Cro) Brilliant red ... 1.00

7.5 Ferdinand Stoliczka (Rich) Light pink ... 2.50

9.3 Festiva Maxima (Miel) Pure white . ... 1.00 
9.2 Florence MrBeth (Sass) Shell pink

each

8.9 Floweret of Eden (Neeley) Mauve pink ...2.200

8.2 Fraicheur (Lem) Creamy white ......... 1.50

8.6 Frances Shaylor (Shay) White ........ 3.00

9.1 Frances Willard (Bra) Blush white ..... 3.00

8.2 Francois Rosseau (Des) Dark red. Early . 3.50

9.3 Frankie Curtis (Vor) A white M. Jules Elie 25.00

8.2 Galathee (Lem) Flesh white ........... 4.00

8.9 Georgiana Shaylor (Shay) Rose pink; large 3.00

George B. Sowersby (Rich) Pink ........ 2.50

8.5 Germaine Bigot (Des) Pale lilac rose ..... 1.00

8.2 Gigantea (Cal) Lilac rose ............. 1.50

8.6 Ginette (Des) Flesh pink; dwarf ....... 2.00

8.2 Gismonda ( Cr) Flesh colored; late ....... 1.50

Gloriana (Neeley) Large pink . . . . . . . . 25.00

Golden Nugget (Pleas) Rosy lilac ...... . 3.00

6.5 Golden Wedding (Pleas) Canary yellow . . 2.00

9.2 Grace Loomis (Saun) White; late ....... 15.00

8.7 Grandiflora (Rich) Silvery pink ......... 1.50

Gretchen (G. \& K.) Light pink ....... . 5.00

8.2 Grover Cleveland ('Ter) Dark crimson; good 1.50

8.2 H. A. Hagen (Rich) Violet rose ........ 3.50

9.1 Hansina Brand (Bra) Pink ..........50.00

8.0 Harriet Farnsley (Bra) Dark crimson.... 1.00

7.9 Helen Wolaver (Bra) Sea shell pink ..... 2. 2.00

Henri Cori (Dor '25) Flesh pink ....... 15.00

8.5 Henry Avery (Bra) White with yel. collar. 4.00

7.7 H. F. Reddick (Bra) Bright dark crimson. 1.50

8.5 Inspecteur Lavergne (Dor) Fine new red . .20.00

7.2 Irma (Cal) Violet rose . . . . . . . . . . . . 1.50

8.3 Isolene (Lem) Pale yellow effect ........ . 3.50

8.9 James Boyd (Thur) Flesh changing white . 5.00

8.7 James Kelway (Kel) Rose white .......... 1.50

8.7 James R. Mann (Thur) Rosy pink ........ 10.00

8.3 Jeanne Gaudichau (Mil) White; late ...... 1.50

9.2 Jeannot (Des) Flesh pink; extra ........ 5.00

8.6 Jessie Shaylor (Shay) Deep cream ....... 5.00

8.5 Jocelyn (Lem) Silvery pink ........... 10.00

9.3 John M. Good (G. \& R.) White ......... 25.00

8.2 John Richardson (Rich) Rose pink ....... 2.00

8.9 Jubilee (Pleas) Flesh white; fine ......... 2.50

8.6 Judge Berry (Bra) Delicate pink; early... 3.50

8.8 Karl Rosenfield (Ros) Dark crimson; good $\$ 1.50$

9.0 Katherine Havermeyer (Thur) Flesh pink. . 15.00

Kelway's David Coral pink ............ 3.50

9.8 Kelway's Glorious (Kel) Creamy white . . . 10.00

8.8 Kelway's Queen (Kel) True. Pink ........ 10.00 


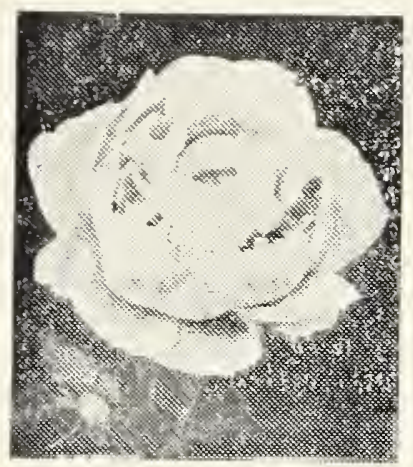

\section{The American Peony Society}

A $\$ 3.00$ membership keeps you posted on the doings in the Peony world. Send check to

Mr. W. W. Cook, Treasurer Clinton, Iowa

King Albert (Kel) Rosy violet .......... 2.50

9.1 Lady Alexander Duff (Kel) Pale pink ..... 2.50

8.2 Lady Beresford (Kel) Blush pink . . . 1.50

8.6 Lady Emily (Pleas) Ivory rose pink ... 3.00

7.3 Lady Gwendolin Cecil (Kel) Lavender flesh 1.00

7.9 Lady Iris (Pleas) Salmon pink ... . 2.00

7.8 Lafayette (Des) Pink with silvery reflex.. 1.50

9.2 La Fee (Lem) Rose pink; beautiful ..... 5.00

9.0 La France (Lem) Violet rose .......... 5.00

8.6 La Lorraine (Lem) Cream white ..... 7.50

8.5 La Perle (Cr) Soft pink; cup shaped ..... 1.00

8.3 La Roserie (Cr) Cream white .......... 1.00

8.1 La Tenderesse (Cr) Milk white ........ 1.00

7.5 La Tulipe (Cal) Lilac white . . . . . . . . 1.00

8.9 Laura Dessert (Des) Canary yellow ..... 5.00

9.9 L Cygne (Lem) Milk white; early ....... 7.50

8.2 Libelleule (Des) Flesh …........ 3.50

8.8 Lillian Gumm (Gumm) Shell pink ....... 7.50

8.1 Livingstone ( $\mathrm{Cr}$ ) Lilac rose ........... 1.00

9.0 Longfellow (Bra) Cherry red; good ..... 3.00

8.4 Lora Dexheimer (Bra) Crimson ......... 2.50

8.7 Lorch (G. \& K.) Cream white .......... 5.00

7.8 Lord Kitchner (Ren) Cherry red ...... 2.00

8.8 Loveliness ( $\mathrm{Hol}$ ) Hydrangea pink ....... 2.50

7.8 Lucy E. Hollis ( Hol) Violet white ....... 1.50

Luella Shaylor (Sha) Creamy yellow center 10.00

8.7 Tuetta Pfeiffer (Bra) Hydrangea pink; early 6.00

9.1 Lucy Shaylor (Shay) White with vel. center 10.00

9.0 Mabel L. Franklin (Bra) Light pink .... . 7.50

8.7 Madelon (Des) Silvery pink; new ....... 10.0

8.7 Magnificent (Bra) Dark red ......... 5.00

9.1 Margaret Veirheller (Wett) White ...... 4.00

8.1 Marguerite Gaudichau (Mil) Shell pink ... 2.50

8.4 Marguerite Gerard ( Cr) Flesh pink ..... 1.00

8.1 Marie (Cal) Lilac White ............. 1.00

8.9 Marie Crousse ( Cr) Shell pink; fine ..... 2.00

R.3 Marie Jacquin (Ver) White; water lily _... 1.50

8.5 Marie Lemoine (Cal) Pure white; late ... 1.00 


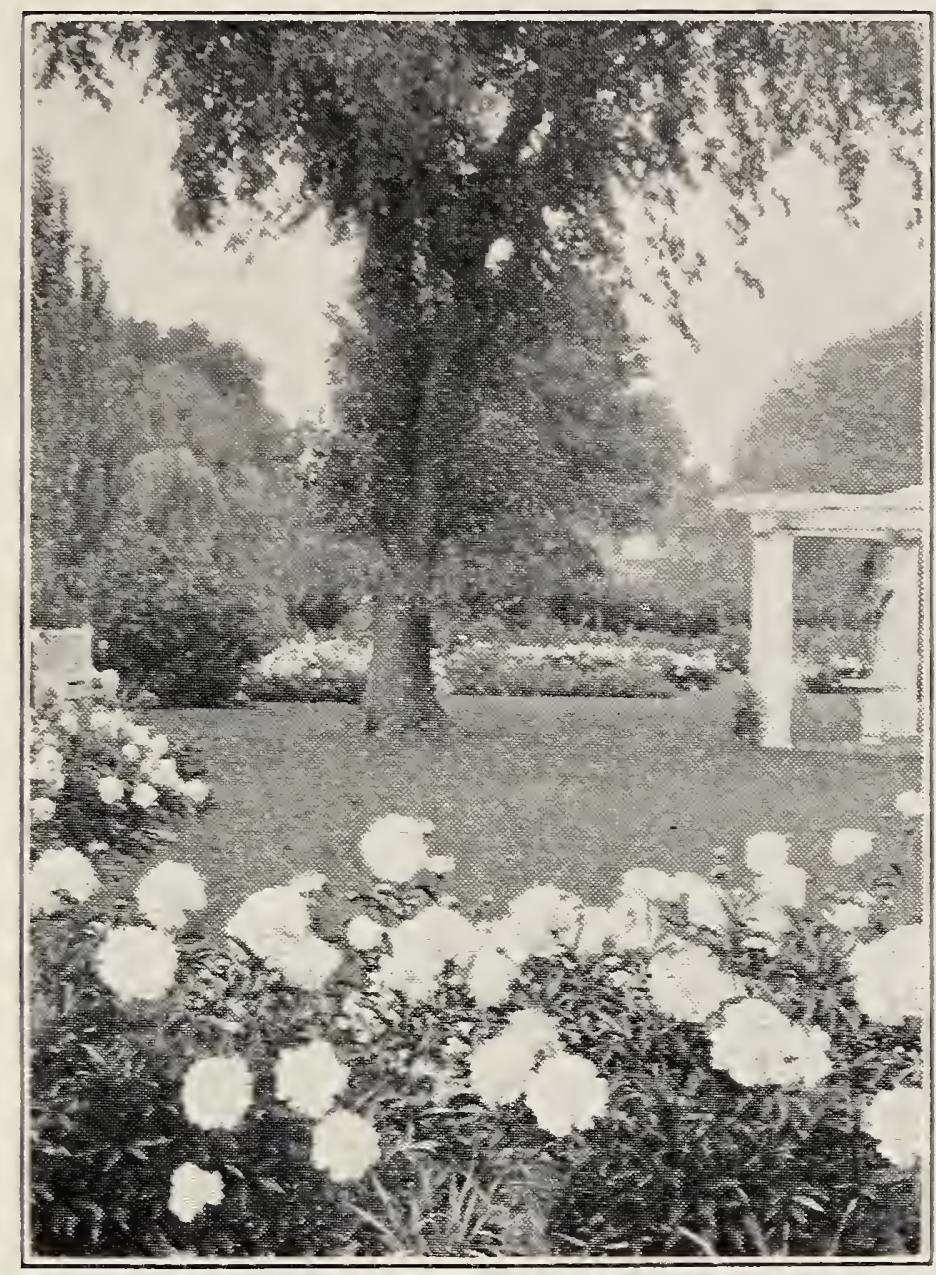

THE PEONY ELWOOD PLEAS

In the foreground. $A$ much admired variety

7.8 Marquis C. Lagergren (Des) Crimson with

silvery border .................. 2.50

each

9.1 Martha Bullock (Bra) Rose pink; large .... 5.00

7.9 Mary A. Livermore ( $\mathrm{Hol}$ ) Rose pink, tipped white

7.5 Mary Baker Eddy (Hol) Rose pink ....... 2. 2.50

8.7 Mary Brand (Bra) Deep red; silky ....... 2.50

8.0 Mary L. Hollis ( $\mathrm{Hol}$ ) Pale lilac ......... 2.00

8.7 Mary P. King (Fra) Coral pink; good ..... 5.00

9.0 Mary Woodbury Shaylor (Sha) Clear pink 5.00

9.0 Matilda Lewis (Saun) Red; fine .......... 15.00

8.5 Maud L. Richardson (Hol) Lilac rose; tall. 2.50

Maurice Materlinch (Kel) Purplish crimson 3.50

7.3 Midnight (Bra) Deep maroon .......... 1.00

8.2 Midsummer Night's Dream (Pleas) Ivory . 2.50

8.7 Mignon (Lem) Amber white; dwarf ..... 3.50

9.0 Milton Hill (Rich) Lilac rose; late ........ 3.50

8.9 Minnie Shaylor (Shay) Semi-double; white 20.00 Miriam (Chase) Rich pink ............ 2.50 


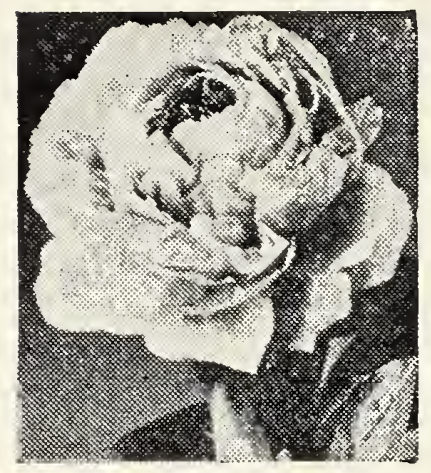

What the Ratings Before the Varieties Mean.

9.5 Almost perfect

9.0 Very flne

8.5 Fine

8.0 Very good

7.5 Good

7.0 Fairly good Riss Emery (Shay) Salmon pink ....... 10.00

7.8 Miss Salway (Kel) Lilac white 3.50

8.2 Mlle. Jeanne hiviere (Rivi) Rose

2.50

Mlle. Louise Delattre (Mil) White ...... 15.00

8.6 Mme. August vessert (Les) Kose pink . . . 1.50 Mme. Bollet (Lal) clear rose . . . . . . . . 1.00

8.0 Mme. Calot (Miel) f lesh ............ 1.00

6.8 Mme. Chaumny (Lal) Lilac rose ......... 1.00

8.2 Mme. De Treyeran (Les) Flesh white ... 2. 2.00 Mme. De Verneville D́lush white . . . . . . 1.00

8.0 Mme. Ducel (Mech) Soft pink bomb ...... 1.00

Mme. Edouard Doriat (Doriat) White ... 15.00

8.4 Mme. Emile Duprez (Kiv) Carmine pink. 3.00

8.5 Mme. Emile Galle (Cr) Lilac white ...... 1.00

8.9 Mme. Emile Lemoine (Lem) Milk white... 1.50

8.7 Mme. Escary (Lem) Creamy white.... . . 10.00 Mme. Francois Toscanelli (Riv) Flesh pink 2.00

8.2 Mme. Gaudichau (Mil) Dark crimson ...... 3.50

7.9 Mme. Geissler (Cr) Rose pink .......... 1.00

7.7 Mme. Guyot (Pail) Cream; yellow center. 3.00

9.4 Mme. Jules Dessert (Des) Cream white... . 3.00

8.5 Mme. Manchett (Des) Silvery lilac ..... 2.00

8.5 Mons. Dupont (Cal) Milk white ......... 1.00

9.2 Mons. Jules Elie ( $\mathrm{Cr}$ ) Deep pink ........ 1.50

8.8 Mons Martin Cahuzac (Des) Dark garnet. 3.00

8.6 Mont Blanc (Lem) Milk white .......... 2.50

8.2 Moses Hull (Bra) Dark shell pink . ..... 1.50

Mr. L. Van Leeuween (Niew) Dark pink. . . 6.00

8.3 Mrs. A. G. Ruggles (Bra) Light pink ..... 4.00

9.6 Mrs. A. M. Brand (Bra. '25) Purest white. 50.00

9.2 Mrs. C. S. Minot (Min) Shell pink and white 20.00

9.3 Mrs. Edward Harding (Shay) Pure white. . 10.00

9.0 Mrs. Frank Beach (Bra) Creamy white . . . 50.00

8.3 Mrs. George Bunyard (Kel) Lilac rose... 5.00

7.9 Mrs. Jennie R. Gowdy (Bra) Pinkish white 3.00

8.2 Mrs. John M. Lewis Dark red; tall ... . . . 3.00 


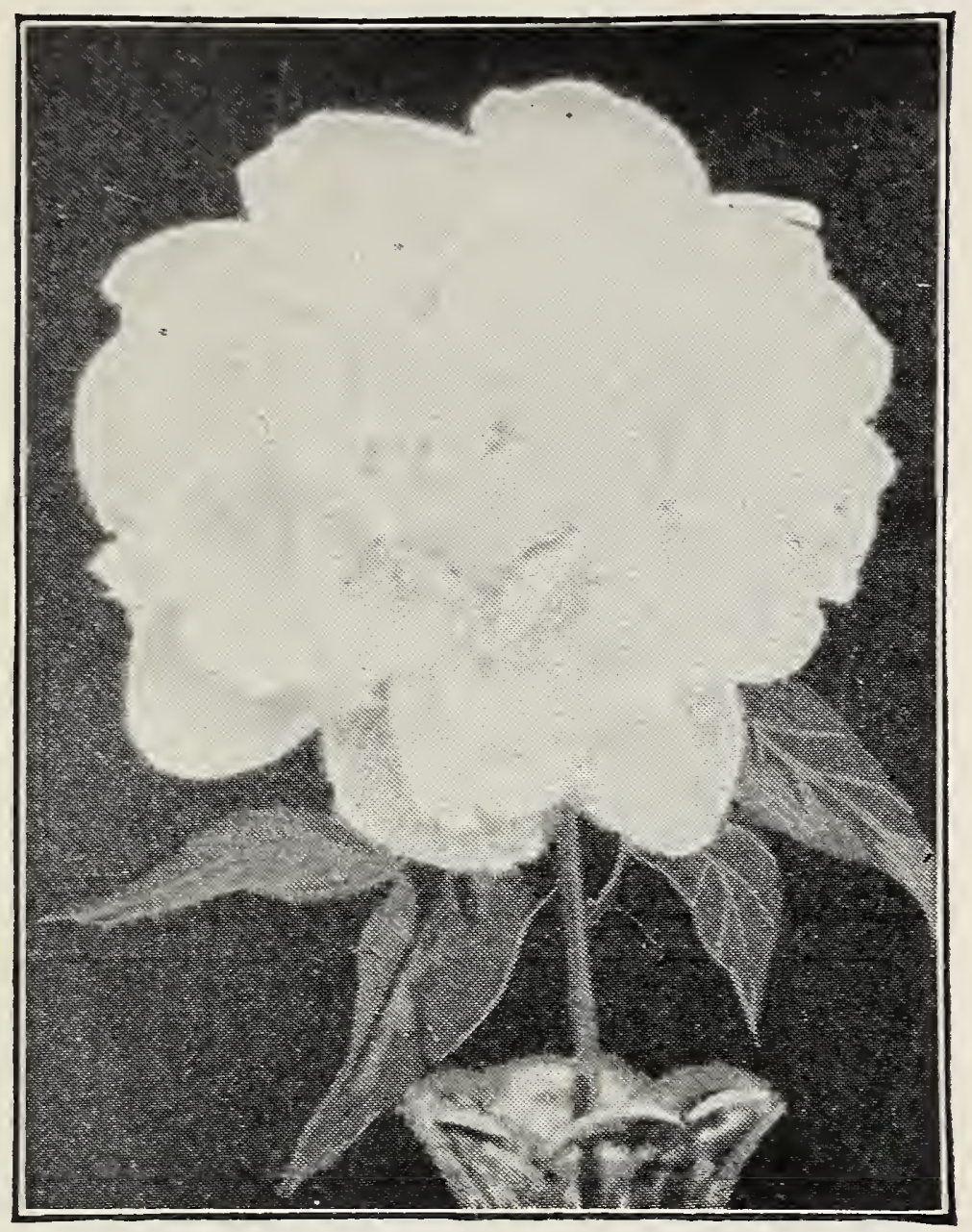

PEONY-MRS. A. M. BRAND

The most beautiful seedling brought out in recent years

each

8.1 Mrs. John Smith Fogg (Hol) Light pink. 2.00

S.8 Mrs. M. P. Clough (Shay) Salmon and white 3.00

9.0 Mrs. Shayor Force (Shay) White ....... 7.50

Mrs. Shuttleworth (Kel) Pale rose ...... 3.00

-.0 Multiflora (Pleas) Silvery pink ........ 1.50

Myrtle Gentry (Bra '25) Rose white .... . 50.00

9.1 Nancy Dolman (Vor' '24) Pink; tall; large. .25.00

Nannette (Doriat '24) Cream white .... 7.50

Nell Shaylor (Shay) White and gold ..... 7.50

n Vim Sroor (Sec) Pure white .......... 10.00

Ninon (1)or) Soft Hesh ............. . 15.00

7.6 Norfolk (Rich) Pale lilar rose ....... 1.00

Nymphe (Har) Dainty China pink ...... 1.50

8.8 Nymphea (Thur) Creamy white ....... 3.00

$\therefore .5$ Octavie Demay (Cal) Pink: fragrant ..... 1.00

8.3 Odalisque (Lem) White and cream ..... 10.00

7.4 Old Silver Tip (Bra) Soft madder rose... 1.50

8.5 Opal (Pleas) Opalescent tints ......... 2.00 


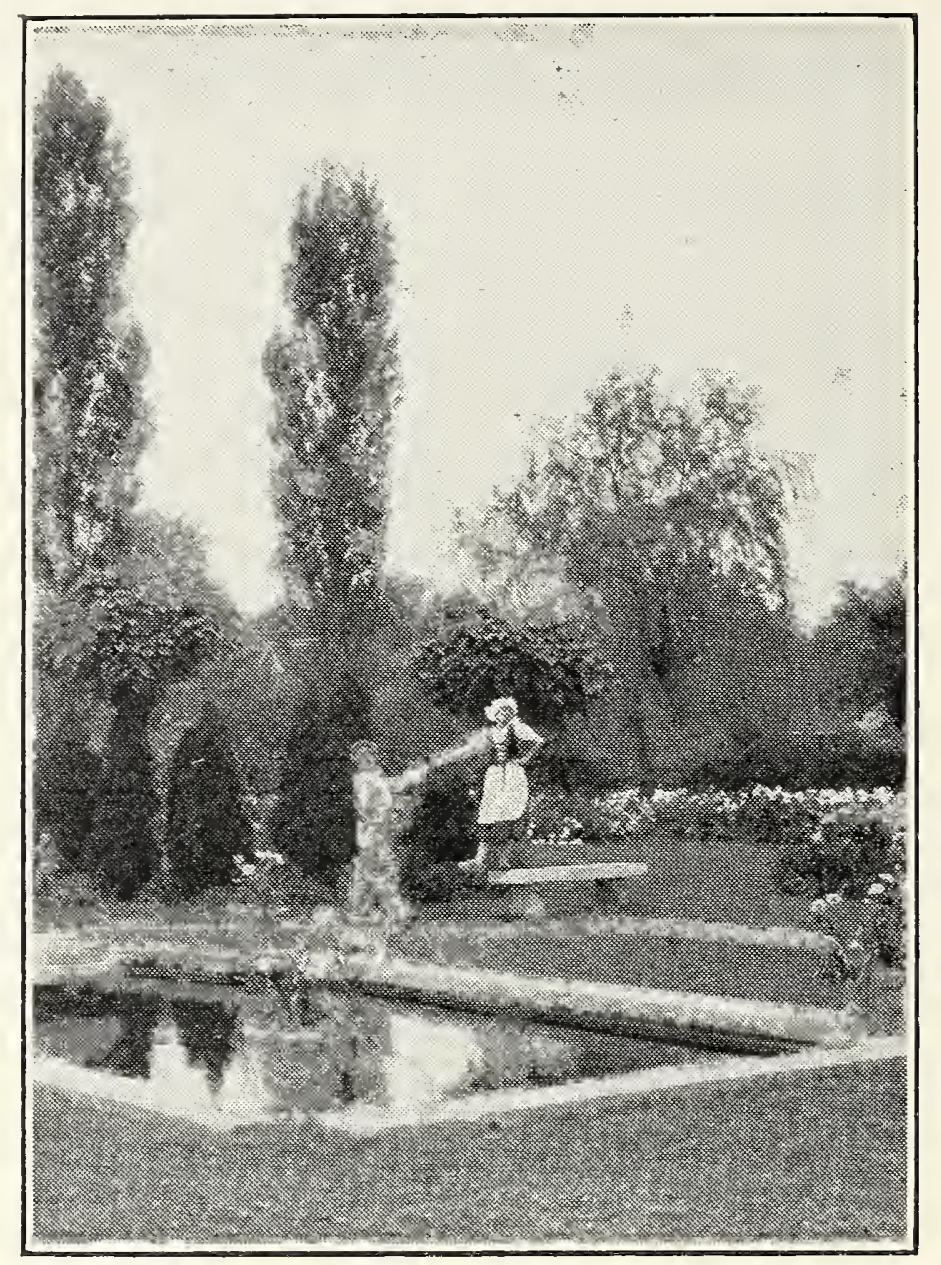

Glimpse of the Wassenberg Gardens

8.0 Opal Iris (Pleas) Salmon pink ........ 2.00

Ostrich Plume White and cream . . . . . . . 1.50

8.2 Pallas Semi rose; early; pink ........ 5.00

8.7 Paradise (Hol) Hydrangea pink ........ 5.00

8.4 Pasteur (Cr) Pink; nearly white ....... 1.50

8.3 Perrette (Des) White, tinged flesh ....... 3.00

8.6 Peter Pan (Hol) Lilac rose ... ....... 2.00

7.6 Petite Renee (Des) Light magenta . . . . . . 1.50

7.7 Philomele (Cal) Pink and yellow ........ 1.00

9.2 Phillippe Rivoire (Riv) Dark crimson . . . . 12.50

8.8 Phoebe Carey (Bra) Lavender pink ..... 6.00

9.0 Phyllis Kelway (Kel) Bright pink ....... 10.00

8.2 Pierre Duchartre ( Cr) Lilac pink ....... 2.00

7.3 Pompette (Des) Pink and silver ........ 1.00

8.7 President Taft (Cal) Delicate pink ....... 1.50

9.3 President Wilson (Thur) Rose pink ....25.00

8.9 Pride of Essex (Thur) Flesh pink. . . . . . . 5.00

8.6 Pride of Paulding (Neeley) .......... 15.00

8.6 Primevere (Lem) Buff and silver ...... 3.00

Princess Olga (Kel) Soft blush ....... 2.50 
each

8.3 Rachel (Lem) Lovely soft pink ....... . 3.50

8.5 Ralph (Pleas) Bright pink .......... 3.50

9.0 Raoul Dessert (Des) Mauve, shaded pink. 7.50 Red Bird (Fra) Red Jap. effect ... . . . . 3.00

8.3 Reine Baronet (Mil) Tyrian pink . . . . . . . 15.00

8.7 Reine Hortense (Cal) Pink . . . . . . . . 1.50

8.4 Reine Marie (Des) Soft pink .......... 3.50

8.0 R. P. Whitfield (Rich) Hydrangea pink . . . 1.50

8.8 Richard Carvel (Bra) Brilliant crimson ... . 3.50

8.6 Rita (Des) Flesh salmon pink .......... 7.50

9.0 Rosa Bonheur (Des) Soft flesh pink ..... 5.00 Rosemary (Kel) Rosy pink .......... 3.00

9.1 Rose Shaylor (Shay) Flushed white . . . . 20.00

8.8 Rosette (Des) Silvery flesh ........... . 3.00

9.0 Rosine (Lem) Late; pale lilac ......... . 5.00

7.9 Ruth Brand (Bra) Lavender pink ........ 1.50

7.5 Samuel Henshaw (Rich) Rose pink ........ 1.50 Samuel Hughes (Kel) Deep amaranth ... 3.50

9.0 Sarah Bernhardt (Lem) Apple blossom pink 2.00

8.5 Sarah Cartenson (Ter) Hydrangea pink . 3.00

9.1 Sarah K. Thurlow (Thur) Pearly white . . . 15.00 Sarah M. Wettengel (Wet) Blush white... 3.00

8.6 Secretary Fewkes (Shay) Cream ....... 6.00

8.5 Shaylor's Dream (Shay) Flesh ........ . 3.50

7.7 Sisters Anne (Bra) Sea shell pink ....... 1.50

9.7 Solange (Lem) Cream coffee color ...... . 3.00

8.2 Sops of Wine (Secor) Dark red ......... 2.50

9.0 Souv De A. Millet (Mil) Amaranthe red . . . 12.50

7.8 Souv. De Francois Ruitton (Riv) Pink .... 2.50

9.1 Souv. de Louis Bigot (Des) Bengal rose ... . 5.00

8.5 Standard Bearer (Hol) Light pink ....... 3.50

8.0 Stephanie (Ter) Delicate pink .......... 1.50

8.4 Strassburg (G. \& K.) Silvery lilac rose... 3.00

7.3 Supreme ( $\mathrm{Hol})$ Pink. Late. . . . . . . . . . . . . 1.50

Sylvia Louise (Kel) Tyrian purple..... . 3.00

8.7 Sylvia (Sau) Pink. Semi-double... . . . . . 15.00

8.2 Suzette (Des) Bengal rose ........... 2.00

8.0 The Gem (Pleas) Dark crimson …... 2.00

9.8 Therese (Des) Soft pink. The best pink ... 3.00

9.1 Thomas C. Thurlow (Thur) Salmon flesh . 20.00

Thurlow's Seedling No. 12 Shell pink.... . 10.00

9.4 Tourangelle (Des) Flesh pink .......... 3.00

7.4 Umbellata Rosea (Des) Pink; early _... 1.00

8.4 Venus (Kel) Delicate shell pink......... 1.50

8.2 Victoire de la Marne (Des) Amaranth ... 5.00 Victory Chateau Thierry (Bra) Bright pink 50.00

7.6 Virginie (Cal) Light pink

Plan to Visit These Farms and Gardens This Year 


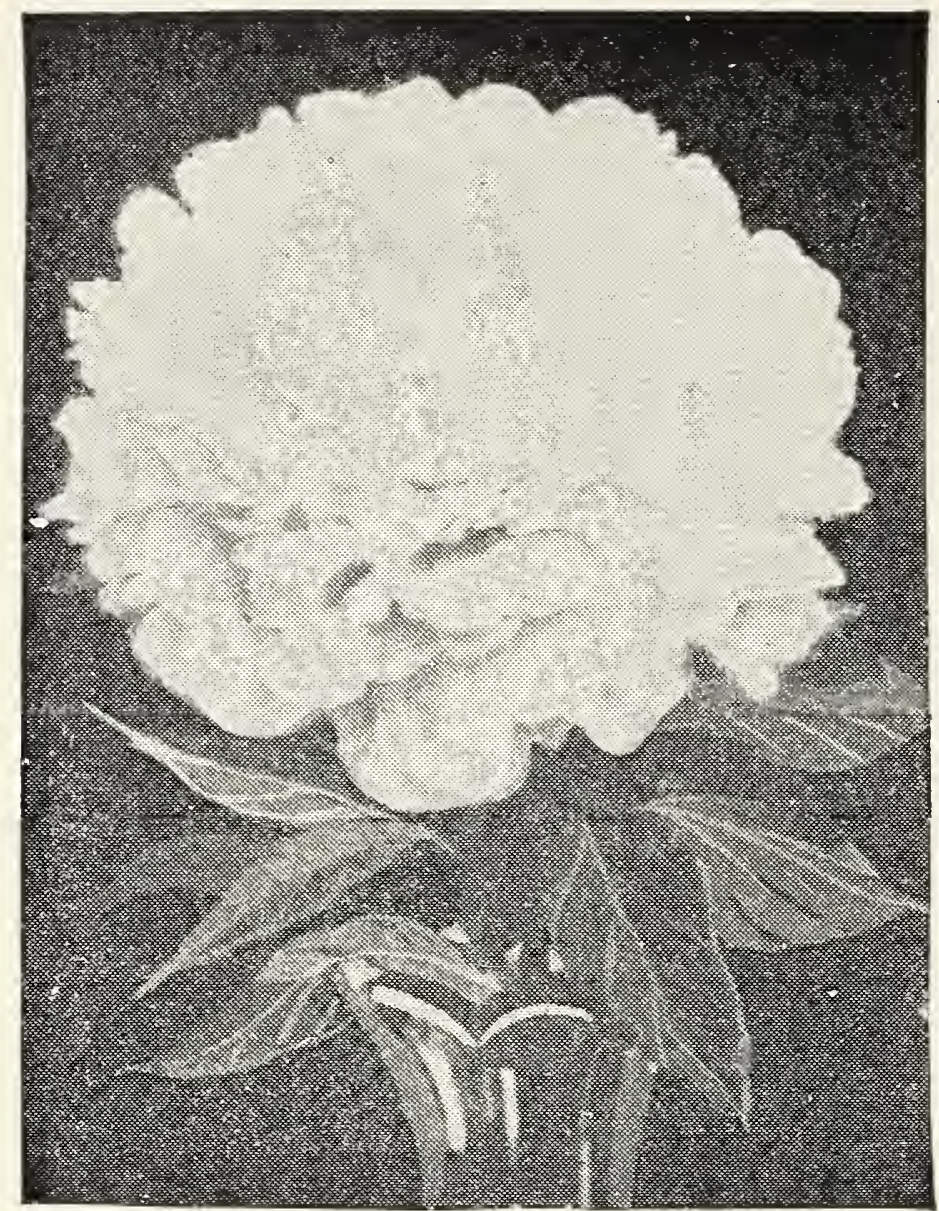

PEONY-MIYRTLE GENTRY

Considered by many as Mr. Brand's greatest production

each

9.3 Walter Faxon (Rich) Salmon rose ....... 3.00
8.2 Weisbaden (G. \& K.) Deep pink ...... 2.50

8.8 W. F. Christman (Fra) Flesh white ..... 5.00

8.4 W. F. Turner (Shay) Dark crimson ..... 4.00

8.8 Wilton Lockwood (Shay) Light pink ..... 7.50 Zest (Hol) Rose pink; late . . . . . . . . . . 1.50

\section{PEONY SPECIES Early Flowering}

Anomala Cut leaves; rose lilac ... . . . \$3.00

8.6 Avant Garde Smoky pink; golden stmens . 5.00 Decora Pallasii Deep crimson; single ... 5.00

8.6 Le Printemps (Lem) Creamy yellow ..... . 5.00 Lobata Cerise . ... . . . . . . 15.00

Officinalis Alba Plena White ......... 1.50

Officinalis Rosea Plena Pink ........ $1.0 n$

Officinalis Rubra Plena Rich red ....... 1.00

Tenuifolia Flora Plena Red ... ..... $\quad 3.00$ 


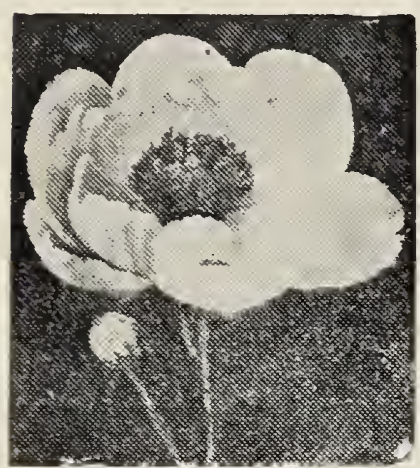

\section{SINGLE PEONIES}

Their early blooming and resistance against storm and wind make them indispensable to any collection or planting.

SINGLE AND JAPANESE PEONIES each

8.1 Akalu (Jap) Carmine red............. 3.00 Akashigata (Jap) Rose ............... 5.00

8.7 Albiflora (The Bride) Single; white guards 2.00

8.3 Alma (Shay) (Jap) Light pink ........ 3.00

8.0 Altar Candles (Pleas) (Jap) Delicate pink 3.00

9.2 Ama-No-Sode No. 1 Light rose pink .... 20.00 Ama-No-Sode No. 2 Wisteria pink ...... 6.00 Apple Blossom (Jap) Flesh pink ....... 6.00 Arlequin (Des) Violet rose ........... 3.00

8.2 Attraction (Hol) (Jap) Tyrian rose .. 2.00

8.9 Auerolin (Shay) Pink guards; yel. center. 5.00

8.5 Black Prince (Thur) (Single) Dark garnet 4.00 Captain Alcock (Kel) (Jap) Pink ........ . 5.00

Caroline (Pleas) (Single) Pink ......... 1.50

Cascade (Jap) White ................ 3.00

8.1 Cathedral (Jap) Deep pink guards ....... 3.50

8.1 Clairette (Des) (Single) White tinged pink 2.00

7.6 Clio (Pet) (Single) Pink; good . . . . . . . . 1.50 Constance (Bern) (Jap) Pink . . . . . . . . 5.00 Dawn (Wallace) (Jap) Pink .......... . 3.00

7.9 Dog Rose (Kel) Delicate pink . ...... . . 5.00

Dr. Edgar Pleas (Pleas) (Jap) Lilac pink . 1.50

Departing Sun (Jap) Rose ........... 3.50

Emma (Jap) Pink ... . . . . . . . . . . 5.00

Emneror of India (Jap) Dark red ...... 3.00

E. St. Hill (Single) Rose pink; large .... . 2.50

8.2 Flashlight (Jap) Tvrian rose . ....... 3.50

7.9 Fugi-No-Mine (Jap) Pure milk white .... 3.50

Fugi-Zoma-Goronn (Jap) Wisteria pink . 6.00

9.2 Fuyajo (Jan) Velvetv mahogany ....... 5.00

Ginko-Nishili ( Tap) Streaked crimson ... 3.00

Flory (Jap) Light tyrian rose ...... 1.50

Golden Nugget (Pleas) (Jap) Rose lilac... 3.00

Fold Mine (Hol) (Jan) Tyrian rose ....... 5.00

8.1 Hana-No-Sota (.Tap) Deen pink ........ 3.50

8.7 Harriet Olney (Bra) (Sin) . . . . . . . 5.00

Hatsugi (Jap) Dark red . . . . . . 1.50

9.0 Henri Potin (Doriat) (Jap) China pink ... 5.00 


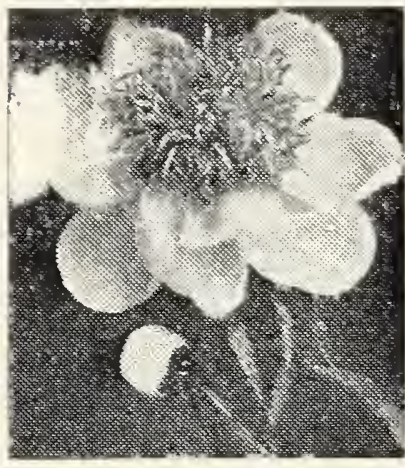

\section{JAPANESE PEONIES}

These are attracting more attention every season, with stocks far behind the demand and will be for a great many years.

SINGLE AND JAPANESE PEONIES each

Hinoke-Sahki (Jap) Violet ........... 3.00

8.3 Innocence (Hol) (Jap) Lilac rose . . . . . . . 2.00

9.3 Isani-Gidui (Jap) Pure white; exquisite. . . 10.00

Kameno-Kerogama (Jap) Clear red ..... 2.50

8.6 King of England (Jap) Carmine ........ 5.00

8.2 Kino-Kimo (Jap) Crimson carmine ...... 3.00

Knight of the Thistle (Jap) Crimson ..... 5.00

Kocho-No-Mai (Jap) Crimson carmine ... 3.00

9.5 Kukeni-Jishi (Jap) Silvery pink ... . . 25.00

Kumagoe (Jap) Crimson pink .......... 1.50

8.7 La Fiancee (Des) (Single) White ....... 2.00

8.6 Le Jour (Shay) (Single) White. Extra ... 5.00

8.4 L'Etincelante (Des) (Single) Carmine .... 3.00

Lord Kitchner (Sir.gle) Rich red; very large 2.00

Lothario (Wet) (Jap) Lavender pink ..... 2.50

7.7 Lucienne (Des) (Sin) White, tinted purple. . 2.00

8.5 Madeline Gauthier (Des) (Single) Pink ... 3.00

8.2 Mafeking (Single) Dark rose pink ....... 2. 2.00

8.7 Marguerite Atwood (Jap) White ........ 7.50

8.5 Marguerite Dessert (Des) White, splashed 4.00

Mellon Knight (Bra) (Single) Rich dark red 3.00

8.6 Mikado (Jap) Dark crimson; extra fine ... 2.50

Minamota (Jap) Purple red; fine ........ 5.00

Minnehaha (Single) Cherry red ......... 3.00

Mischief (Auten) (Single) Pink; good ... 6.00

Miyo-No-Hana (Jap) White; lovely ..... 10.00

Moonbeam (Sin) Opal tints ........... 5.00

Mrs. Laura Coates Reed ( $\mathrm{Hol}$ ) Dark garnet 3.00

Nabunshki (Jap) Streaked carmine...... . 1.50

8.6 Nellie (Single) Pink blush .......... 3.50

Noon Day (Jap) Rose; very attractive ... 5.00

Nymphe (Des) (Single) Dainty pink ..... 1.50

Nymphe (Har) China pink . . . . . . . . . 1.50

Oahio-Kun (Jap) ................. . . 3.00

6.9 O'Fugi (Jap) Flesh guard petals ....... 2.50

8.2 Ohirama (Jap) Bright pink .......... 4.00

Oki-No-Nami (Jap) Hydrangea pink .... . 3.00

Onahama (Gumm) (Jap) Dark red .......10.00

BE SURE TO INCLUDE A JAP OR SINGLE 


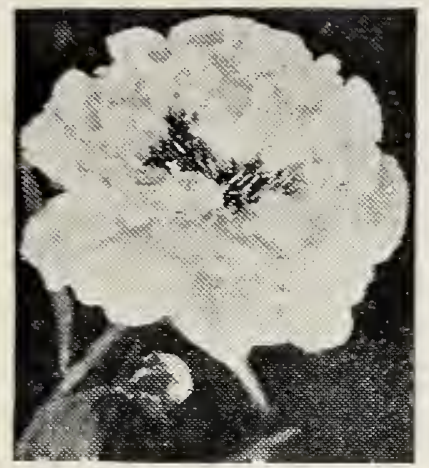

\section{NEW PEONY MANUAL}

Just issued by AMERICAN PEONY SOCIETY

The last word on all phases of the Peony. Every Peony Iover Should have a copy

$\$ 6.00$ POSTPAID

Send us your check

SINGLE AND JAPANESE PEONIES

Ostrich Plume (Pleas) White guards ..... 1.50

8.4 Perle Blanc (Des) (Single) Pure white... 2.50

Perle Rose (Des) (Single) China pink .... 2.00

Pocahontas (Pleas) (Single) Rosy red .... 1.50

8.7 Princess Duleep Singh (Jap) Rose pink . 5.00

Princess Matilda (Des) (Single) China pink 2.00

8.9 Pride of Langport ( $\mathrm{Kel}$ ) ( Sin) Peach pink. . 6.00 Pure Love (Kel) Shell pink ............ 3.00

Queen of Pleasance (Pleas) (Jap) Rose ... 1.50

8.7 Rashooman (Jap) Crimson guards ....... 5.00

Rose of Nippon (Jap) Deep rose; different 3.00

7.7 Sagoromo (Mil) (Jap) Salmon guards ... 10.00

8.2 Sieru Somae (Jap) White and sulphur... 3.00

Shiro-Sangai (Jap) White guards ...... 3.50

8.: Snow Wheel (Jap) White ............. 5.00

Sochi (Jap) Deep rose ................ 7.50

9.0 Soma-Ganoka (Jap) Dark red guards .... 6.00

7.8 Stanley (Single) Dark Crimson ......... 2.00

Sylvia (Saun) Pink; water lily effect . . . 15.00

Tamate-Boku (Jap) Best pink ... . . . . . 25.00

7.8 The Moor (Single) Purple garnet ....... 1.50

8.9 Tokio (Jap) Clear pink ............ 7.50

Toreador (Jap) Red; late; very tall .... . 2.00

9.0 Toro-No-Maki (Jap) White ............ 10.00

8.0 Torpilleur (Jap) Carmine purple ........ 5.00

7.3 Veloutine (Single) Tyrian purple ......... 1.50

Venise (Des) (Single) Brilliant pink ...... 1.50

8.8 Vera (Gumm) (Single) Dark red ... . . . . . 10.00

7.6 Vesuve (Des) (Single) Dark red ......... 1.50

White Japan (Single) White ........... 2.00

9.0 White Lady (Single) White . . . . . . . . . . 10.00

White Swan (Single) White ........... 3.00

Whitleyi Major (Single) White ........ 1.50

Wild Rose (Pleas) (Single) Pink ........ 1.50

Yamato-Tsukasa (Jap) Bright rose ..... 3.50

7.7 Yeso (Jap) White ................. 2.50

MAKE A RECORD OF YOUR PLANTING 


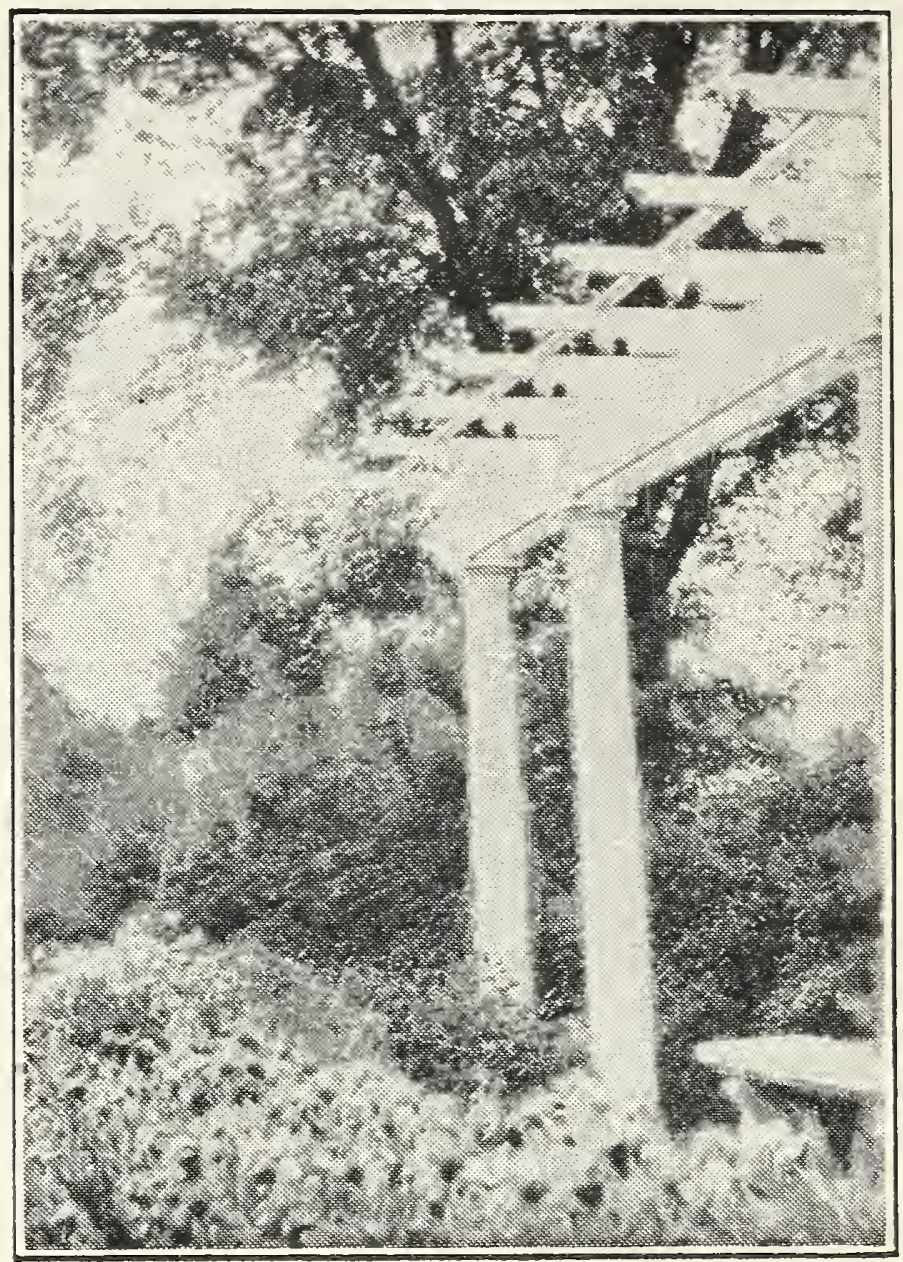

WASSENBERG GARDENS

\section{IRIS}

7981 Abenda (Per) Pure white; fine form

8382 Afterglow (Stur) Greyish lavender

New Alabaster (And) Pure white; fine

each

$\$ 1.00$

8687 Alcazar (Vil) S. violet; F. purple; tall . .35

New Alcyon (Cay) ................... 3.00

8988 Aliquippa (Hall) Pale Massicot yellow. 3.00

8384 Allies (Vil). Smoky wine ........... 7.50

85 85 Alvarado (Mohr) Tall; rich deep purple . 1.00

7874 Amas (Wild) S. blue; F. violet ..... .25

8291 Ambassadeur (Vil) S. bronze; H. maroon .50

8888 Amber (Dykes) Striking deep yellow . . . 3.50

7879 Ambigue (Vil) S. smoky; F. purple ... .35

New Amerind (And) Metallic bronze ....... 5.00

New Andromede (Vil) New and rare ....... . 10.00

New Antares (Vil) Yellow blends; new ...... 3.00

Now Antonio (IIont) Intcne blue violct .... 1.00

\section{ALL RETAIL SHIPMENTS PREPAID}


each

8685 Ann Page (Hort) Pale lavender blue self 1.00

s0 77 Anna Farr (Farr) White and blue edge. . .75

85 84 Anne Bullen (Hort) S. lav.; F. vel. pur. 1.50

7575 Anne Leslie (Stur) S. white; F. purple $\quad .50$

8188 Aphrodite (Dyk) S. \& F. bright lilac pink 2.00

8587 Apache (Farr) S. copper pur.; F. crim.. 3.50

7981 Archereque (Vil) S. \& F. purple .... $\quad .35$

New Arcturus (Vil) New French variety ... 3.50

84 88 Argynnis (Will) S. yellow; F. dark car. .75

New Argos (Vil) New; medium dark ..... 3.00

New Ariel (Murr) Soft Harebell blue 2.00

8484 Arlington (Simp) Rich purple effect; tall 1.50

New Arzani (Stur) Red toned; three ft. . . 5.00

9288 Asia (Yeld) S. silver; F. raisin purple. 1.50

New Athaniel (Mil) ............... 3.00

7377 Aurea (Jacq) S. \& F. chrome yellow... .25

7273 Autocrat (Clev) S. blue; F. dark blue . .35

7477 Autumn King (Sass) Blooms spring \& fall 1.50

80 Avril (Den) S. violet; F. blueish purple. 1.50

7774 Azure (Bliss) S. lavender blue; F. purple .25

8382 Balboa (Mohr) Red-violet bicolor ..... 2. 2.03

9089 Ballerine (Vil) S. violet; F. deeper .... 1.00

8685 Bandolero (Mohr) Distinct light blue; 3 ft. 1.00

7878 Baronet (Stur) S. blue; F. deeper blue. . .35

8278 Beau Ideal (Sass) White plicata; large. . .75

7678 Ben Bow (Bliss) Dark bluish violet .... .50

8987 Bertrand (Bliss) Dominion seedling ... 3.50

7872 Black Prince (Per) Dark violet-purple. 1.00

67 Blue Boy (Fos) S. \& F. rich purple ... . .25

74 Bluet (Stur) Blue tone; free blooming. . .25

8179 Bollingbrake (Hort) Milky white ..... 2.00

8182 Brandywine (Farr) Silvery violet effect 1.50

New Bravura (Mohr) Deep rosy lilac; 40 in.. 1.50

9190 Bruno (Bliss) S. bronze; F. red purple . 5.00

New Buto (Sass) Deep blue purple . 7.50

8480 B. Y. Morrison (Stur) S. violet; F. purple .50

7780 Camelot (Bliss) White, edged violet .25

9691 Candlelight (And) Pale pinkish lavender 5.00

84 8.3 Canopus (Bliss) S. lavender; F. Pan. viol. 1.50

7172 Caprice (Vil) S. rosy; F. rosy red .... 25

9290 Cardinal (Bliss) Lavender and prune . . 7.50

8483 Caroline E. Stringer (Sass) Pale pink. 1.50

8282 Cassandra (Per) S. rose vio.; F. dk crim. 1.50

New Cassiopee (Vil) New; 3 ft.; med. late. 3.00

727 Cavalier (Clev) S. blue; F. purple ..... .25

New Caylus (Vil) Redder Alcazar ........ 2.50

66 72 Celeste (Lem) S. \& F. azure blue ..... .25

8586 Centurian (Bliss) Dominion seedling ... 5.00

PLANT QUANTITIES FOR COLOR EFFECT 


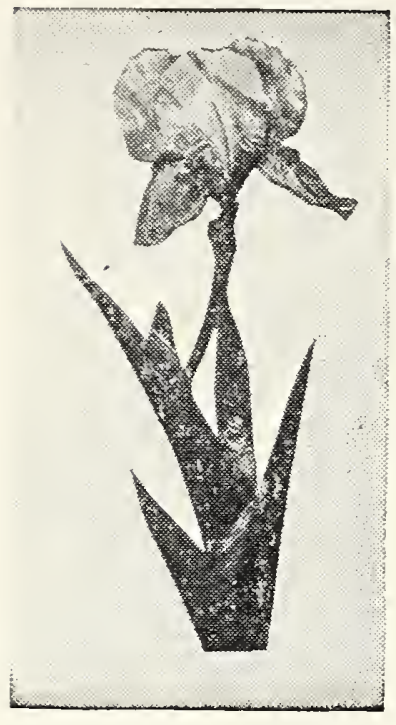

\section{AMERICAN IRIS SOCIETY}

\section{Your Membership Solicited}

You will enjoy the Bulletins as they will keep you posted on the merits of the newer varieties.

Membership $\$ 3.00$

Send check to

J. B. WALLACE, Jr., Sec.

129 Church St. NEW HAVEN, CONN.

8285 Chasseur (Vil) Tall; straw yellow ..... 1.50

8889 Chartier (Hall) New; tall; white ...... 3.50

Chester J. Hunt (Farr) S. blue; F. marine .25

8585 Citronella (Bliss) Gold and maroon; fine 2.00

8080 Cluny (Vil) S. lilac; F. dark lavender. . .35

7678 Col. Candelot (Mil) S. brown; F. dk crim. .25

8885 Concohbar (Bliss) Purple bicolor ..... 3.00

New Coppersmith (Shull) Copper color sheen 5.00

8983 Conquistador (Mohr) Deep mauve; tall 1.50

6975 Cordun Blue (Stur) Blue toned ....... .35

8284 Corrida (Mil) Sky blue self .......... .35

7576 Cretonne (Bliss) S. bronze; F. red-mar. .35

8585 Crusader (Fos) S. bluish; F. dark bluish .50

7679 Dalila (Denis) S. white; F. red-purple . .35

6872 Dan'l Lesuer (Den) Mauve and violet. . .25

80 81. Daphne (Bliss) S. white; F. purple .... $\quad .50$

7175 Dawn (Yeld) Sulphur yellow ........ .25

Dejah (Per) S. silvery blue; F. mauve b. 1.00

7778 Dejazet (Vil) S. bronze; F. violet

8080 Delicatissima (Mil) Delicate lilac

8483 Delight (Stur) Plicata; choice coloring . 2.50

69 Demi-Deuil (Denis) Veined mauve ..... $\quad .35$

7873 Dionyza (Hort) Very lovely lavender .... 2.50

New Distinction (Cay) Soft rose . ......... 3.00

9391 Dolly Madison (Wil) A glorified Quaker Lady ..... . . . . . . . . . . . 17.50

8882 Dominion(Bliss) S. Dauphins blue; F. pur. 5.00

7678 Dora Langdon (Bliss) S. lilac; F. reddish .35

75 Dorothea (Cap) Pearl grey; early ..... . .25

8484 Dream (Stur) Light pink; very popular .50

7577 Du Guesclin (Bliss) S. coppery; F. violet .35

6787 Dusky Maid (Bliss) S. buff; F. purple.... .35 
each

7779 Eckesachs (Gok) S. lav. F. purple violet .35

7383 Eclaireur (Cay) White ruffled; F. viv. rose .50

7881 Ed Michel (Verd) Deep red violet ...... .50

6281 E. J. Jenkins (Bliss) Pale steel blue... .50

7576 Eldorado (Vil) Bronze tons ......... . . .25

8887 Elaine (Shull) Yellow and violet blend . 3.00

8184 Ember (Stur) Violet-red ............ 2.00

New Etoile du Matin (Vil) Blend; new ...... 3.50

8587 Evadne (Bliss) S. rose violet F. red... 2.00

New Eventide (Sass) Slatey lavender ..... 2.50

7678 . Fairy (Ken) White and soft blue ...... 25

8080 Flammenschwrert $(G \& K)$ S. bright yellow; F. red ................. .75

Florentina (Alba) Early white ...... .25

8084 Folkwang ( $\mathrm{G} \& \mathrm{~K}$ ) Lustrous pink . 1.00

7879 Forsette (G \& I ) Dark lavender; $3 \frac{1}{2} \mathrm{ft} .1 .00$

New Fra Angelico (Vil) Sunset effect; fine. . 5.00

8995 Freida Mohr (M.M.) Pink bicolor; large 7.50

7676 Fryers Glory (Fry) S. bronze; F. maroon .35

7380 Furstin Lonyay (G \& K) S. lilac; F. wine .50

7681 Garden White (Stur) ............. 3.00

9088 Geo. J. Tribolet (Will) Dark red purple 3.00

8385 Geo. Yeld (Per) S. apricot; F. rose crim. 1.50

83 Georgia (Farr) Cattleya rose; good .. . $\quad .50$

9190 Germaine Perthius(Mil) S. vio. pur. F. vio. 4.00

7068 Glitter (Bliss) S. yellow; F. brown .... . .35

8786 Glowing Embers(Stur) S. lav.; F. dah. pur 2.00

7576 Gold Crest (Dyk) Bright violet blue.... .35

New Goldenrod (Row) Deep yellow; tall ... 1.00

71 Goliath (Cay) S. violet; F. purple ...... .25

8687 Gold Imperial (Stur) Chrome yel.; good 1.50

6868 Gov. Hughes (Fray) S. violet; F. deeper .25

78 Grevin (Vil) Violet and yellow ........ .25

8381 Halo (Yeld) S. violet; F. bluish violet.... .50

7982 Harmony (Dykes) Dark purple self ... 1.00

7777 Harpalion (Per) S. lav.; F. lav. blue; tall 1.00

8283 Harriet Presby (Pres) Tall rose lilac . 1.50

7074 Her Majesty (Per) Rosy pink ......... . . 25

8482 Hesperia (Wil) Brown effect ......... 1.50

New Horizon (M.S.'25) Light blue lav: 42 in. 3.00

9080 Hypolyta (Hurt) Clear lav.; fine; early .75

New Hussard (Vil) Self blue ............ 1.00

New Ideal ( $G$ \& $K$ ) S. blue; F. royal blue ... 1.00

Iduna ( $G \& \mathrm{~K}$ ) Cream and black purple 1.50

Igouf (Mil) Blue and violet .......... .25

8384 Imperator (Cay) Imp. Seminole; good. . 1.00

8080 Iris King $(G \& K)$ S. yellow; F. maroon .25 


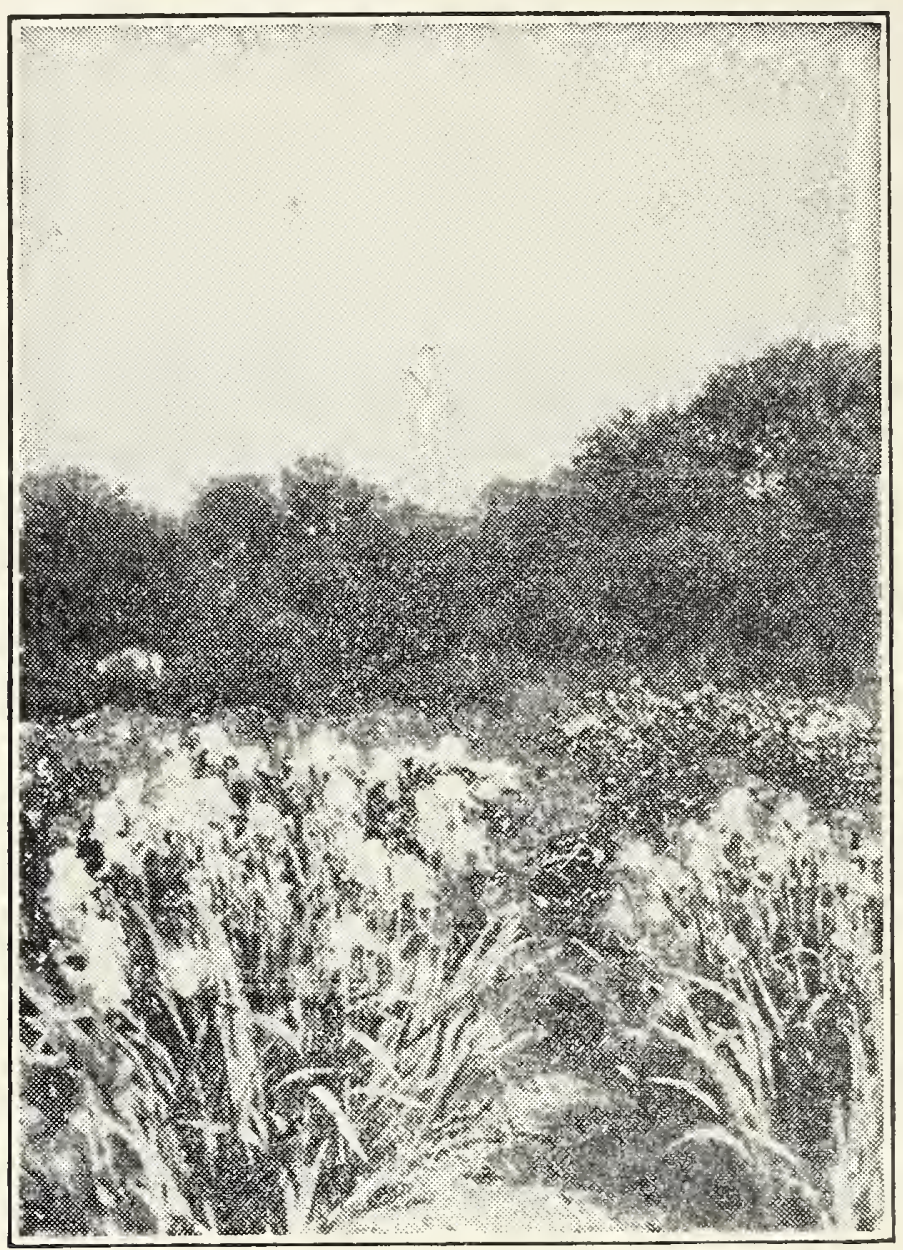

ROWS AND ROWS OF PEONIES AND IRISES We know you will enjoy seeing the varieties you have heard and read so much about.

Every plant labeled.

7783 Isis (Bliss) China-blue ........... 1.00

8384 Isolene (Vil) Lilac and purple .............

8280 Jac. Guillot (Cay) Soft lavender violet. 1.00

8381 Jean Chevreau (Cay) Brown syots .... $\quad .75$

8584 Jubilee (Sass) Ruffled; cream dotted ... 2.00

8585 Julia Marlow (Shull) S. Chinese violet; F. purple

7881 Juniata (Farr) S. \& F'. Clear blue; tall .25

76 73 Kathryo fryer (Fay) S, vinlet: F' mar. 50

8584 Kestral (Mor) Reddish monle bicolor. 5.00

74 Kharput S. violet: F. mole 25

8583 King Kart (Sass) Dotted pink and reddish 2.00 7476 Kynsna (Bliss) S. vellow; F. red brown .25

7580 Kochii (Wild in Italy) Claret and purple .25

New Labor (Cay) Elcetric blue sheen … 7.50

80 8.3 Lady Byn: (Pliss) Clear pale lav.; late 1.00 \&6 87 L'Aig"on (Shull) A mbassadem type;redder 6.00 
each

8583 Le Grande Ferre(Cay) S. Grey; F. reddish 2.50

8583 Le Neige (Verd) Good white .......... .35

8888 Lent A.Williamson(Wil)Vio. \& pur.;early .35

S7 88 Leonata (Hort) Pale pur. bicolor; large 3.00 Leota (Fray) White; early .............35

3682 Leverier (Ien) S. vio.; F. pansy violet . . 1.00

7679 Lohengrin ( $\mathrm{G} \& \mathrm{~K}$ ) Cattleya rose ..... . .25

8483 Lona (Sass) Soft buff; peppered violet. . 1.50

8483 Lord Lambourne (Per) Dusky red effect 2.00

8878 Lord of June ( eld) Lavender blue; large .75

7378 Loreley ( $G$ \& $K$ ) S. yel.; F. marine blue .25

8.5 81 Louis Bell (Den) Black vio.; darkest of all 5.00

8080 Mady Carriei๋ (Mil) Greyish lav. blend .35

7575 Magnate (Stur) Red purple tones ...... .25

8785 Magnifica ( $V$ iI) Violet and reddish violet 1.00

8887 Majestic (Bliss) Dominion seedling; good 4.00

7880 Ma Mei (Cay) White: frilled blue ...... .35

3281 Maori Princess(Shull) S.yel.;F.burnt lake 1.50

6465 Margaret Moor (Bliss) Reddish lilac .. .25

8181 Marjorie Tinley (Per) Lav. blue bicolor 1.50

8281 Marsh Marigold (Bliss) Variegata S. yel.;

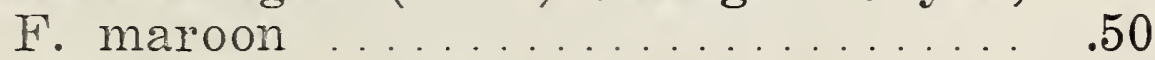

8890 Mary Barnett (Cumb) Imp. Princess Bea. 5.00

7272 Iary Garden (Farr) Dotted Maroon .... . .25

8485 Mary Gibson (Per) Rose bronze blend; 2.00

8181 Mary Orth (Farr) Two shades blue violet .50

7272 Mauvine (Dean) Mauve self.......... .35

7273 May Rose (Clev) Pink tones .......... .25

8487 Medrano (Vil) Dark smoky claret ...... .75

Memory (Yeld) Pale mauve and gold.. .50

7977 Merlin (Stur) Mauve and purple ..... .35

8582 Mentor (Per) S. rosy lav.; F. crim. pur. 1.50

8383 Mestor (Per) S. violet blue; F. pansy vio. 2.00 Midgard (Sass) Yellow pink blend..... 5.00

8180 Mid-West (Sass) Ruffied; dotted purple .50

8687 Mildred Presby (Farr) S. white; F. deep violet

1.50

7573 Minnehaha (Farr) White; mar. markings .25

7977 Miranda (Hort) Violet self; early ...... .25

7774 Mistress 1 ord (Hort) Red purple ...... 1.00

8884 Mile. Schwartz (Den) Mauve; 31/2 ft. . .50

8586 Mme. Abel Chautney (Cay) Gold; Rose ash color .................. 1.00

7373 Mme. Boullet (Den) Dotted mauve ... . .25

8483 Mime. Cheri (Stur) Violet pink ....... .50

6970 Mime. Chevreau (Lem) White; frilled blue .25

8180 Mme. Chobaut (Dn) Buff and wine dotted .50 


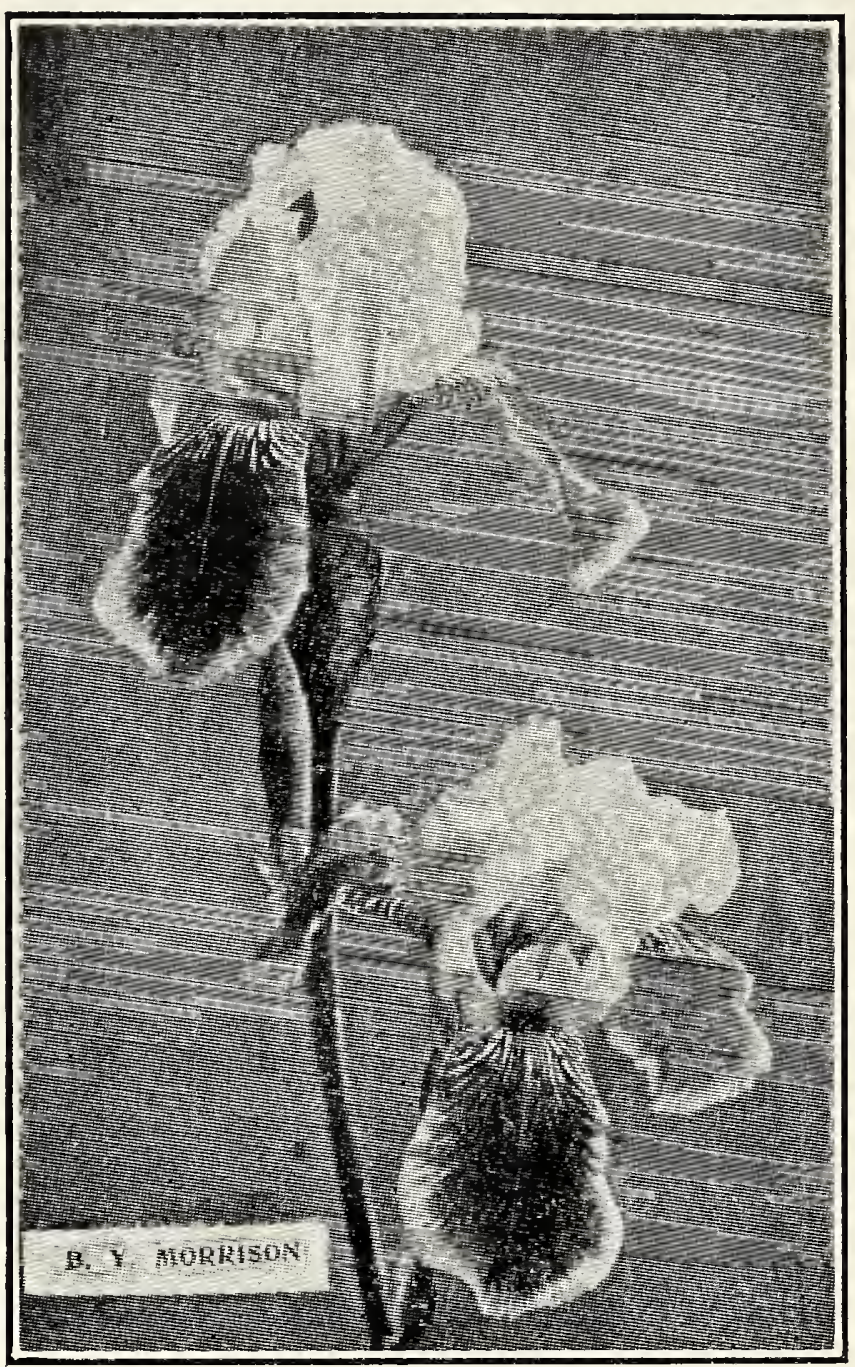

IRIS-B. Y. MORRISON

A most striking and beautiful variety. One of the best in its class

each

7466 Mme. de Sevigne (Den) Purple markings .25

7979 Moliere (Vil) Dark violet ............ .50

7477 Monsignor (Vil) S. violet; F. purple .... .25

7878 Montezuma (Farr) Dotted brown ...... .25

8584 Moonlight (Dyk) Fragrant; silvery white 2.00

6974 Moonstone (Clev) Pale blue .......... .50

9191 Morning Splendor (Shull) Red purple.. 1.50

8484 Mother of Pearl (Stur) Pearl tints ..... .50

7174 Mrs. Alan Gray (Fos) Mauve and violet .25 Mrs. Cuthbertson(Per) S.rose;F.rose crim 1.50 Mrs. Emma Nevada (Per) Tall; pale blue and crimson

7780 Mrs. Hetty Matson (Per) S. rose lilac;

F. crimson purple

8083 Mrs. H. F. Bowles (Per) Old gold and brick 1.50 


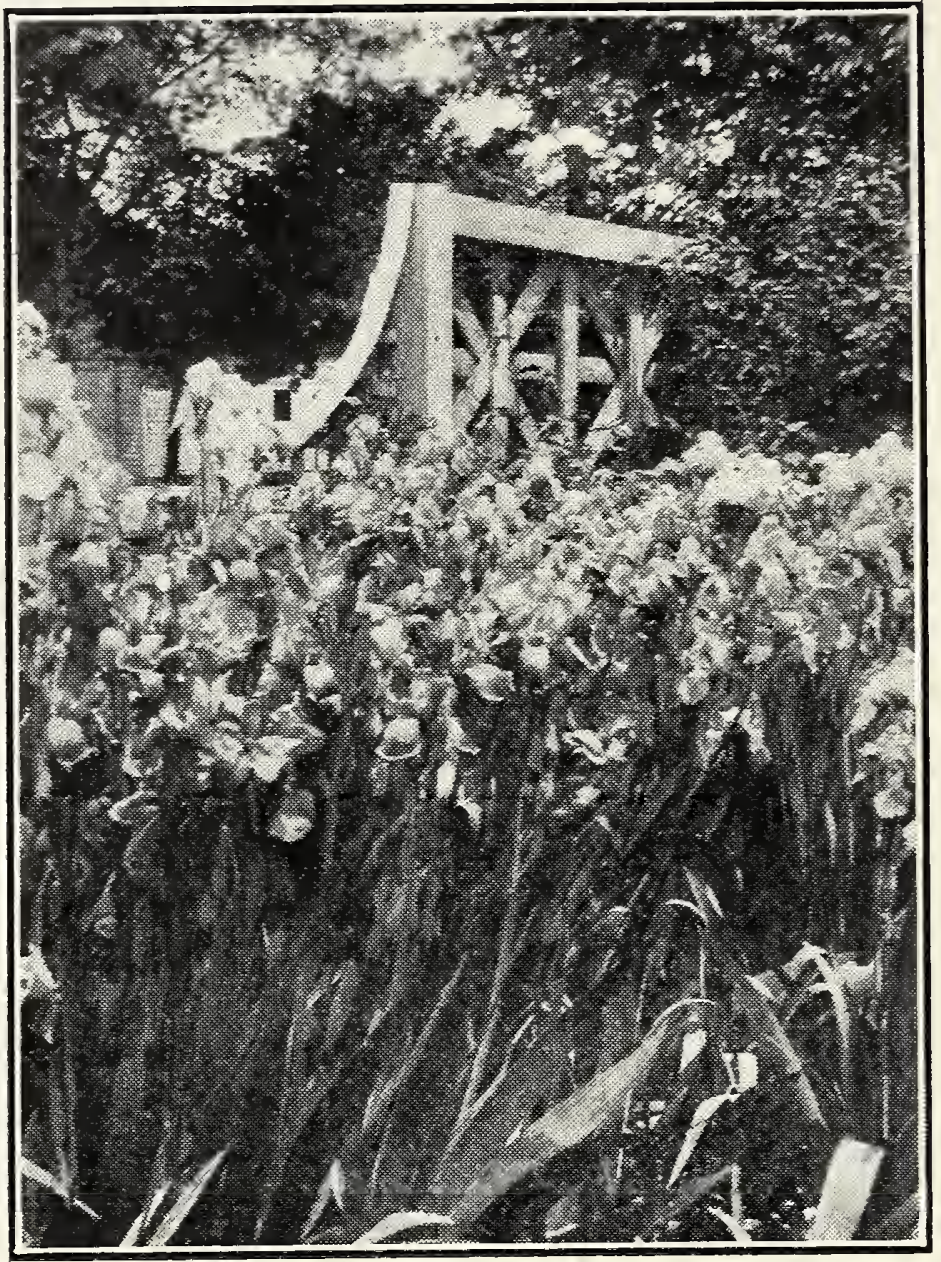

IRISES EN MASSE

Showing how Irises give beauty and color to the garden.

8888 Queen Caterina (Stur) Pale orchid ... $\quad \begin{array}{r}.50 \\ 6\end{array}$

6768 Queen Elinor (Hort) Pink toned ...... .35

Queen Chereau (Bal) S. \& F. white; suffused blue .................. 1.00

7174 Queen of May (Sal) Lilac pink; early.. .25

7474 Raffet (Vil) Dark blue purple; late ... .35

7979 Ragan (Hort) Violet blue bicolor : 3 ft. . . .25

8182 Rajput (Stur) Bright violet self ...... 1.50

Ramona (Mohr) Violet; flushed bronze . 1.00

Red Cloud (Farr) S. bronze; F. maroon. . .25

6661 Red Cross (Clev) Lavender and red. .50

New Rhea (Will) S. lobelia vio.; F. pansy vio. 2.50

E3 87 Rheingauperle (G\&K) Soft orchid pink 1.50

8283 Rhein Nixie (G\&K) S. white; F. blue; late .25

7679 Rheintraube (G\&K) S. vio. blue; F. pur. .50

67 Rhoda Pink and rosy mauve ...... . . .25

8383 Robt. W. Wallace (Per) Glor. Archeveque 2.50 


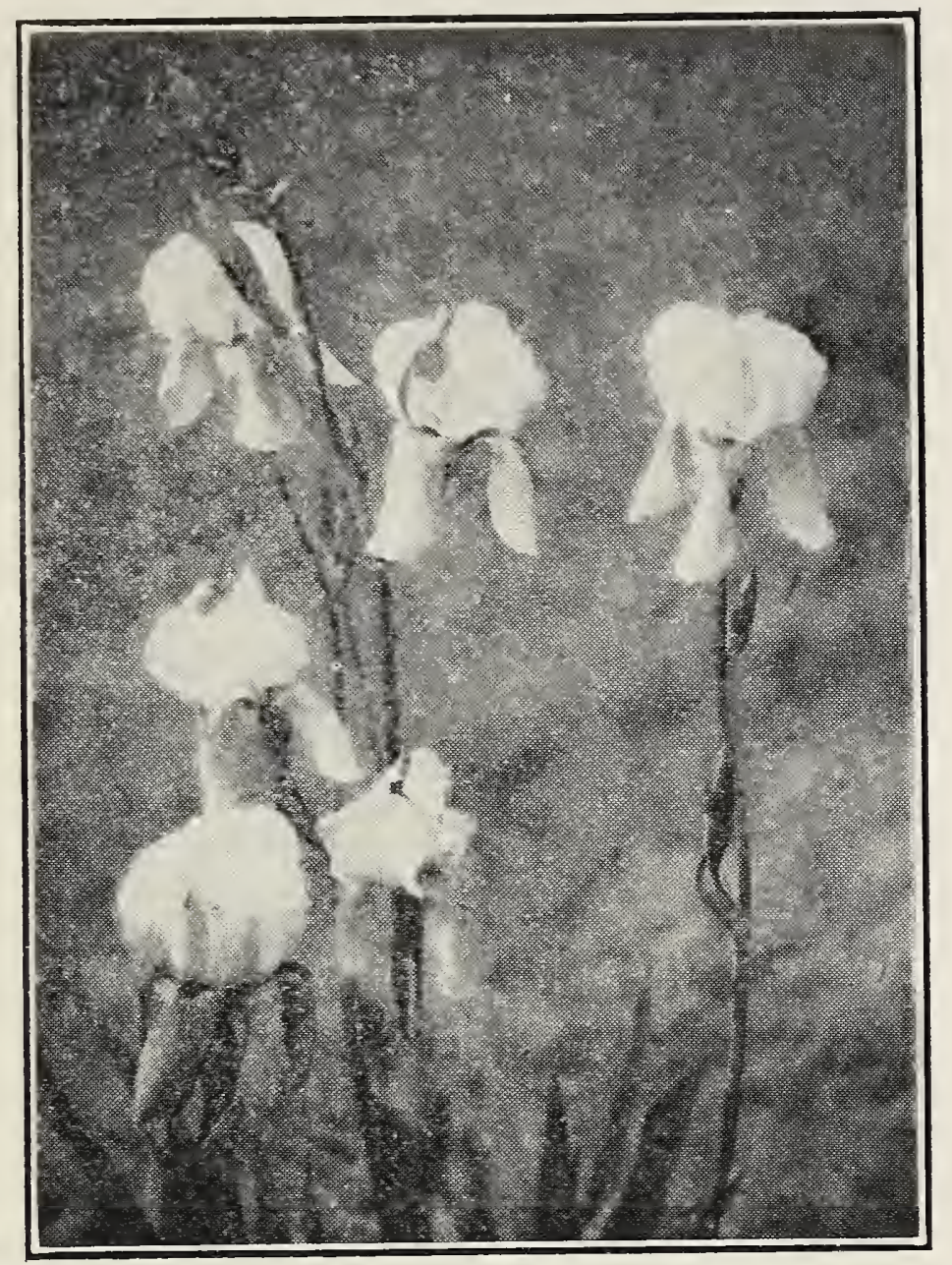

\section{IRIS-LORD OF JUNE}

Admired By All. Exceptionally Fine

6972 Rosalind (Bliss) Pink mauve; tall; late. .50

8283 Rose Madder (Stur) Bright rose madder 2.00

69 Rose Unique (Farr) S. \& F. violet rose. . .25

7478 Rota (G\&K) Mass effect red; $21 / 2 \mathrm{ft} . \ldots . .1 .00$

7883 Rubyd (Dyk) Improved. Ed Michel.... .50

7778 Salonique (Cay) S. white; F. velvet pur. .50

7376 Saul (Den) S. yellow; F. maroon..... . .25

8383 Seminole (Farr) S. red tones ......... .50

8786 Sequoiah (Shull) S. lilac; F. velvety pur. 6.00

8484 Shekinah (Stur) Pale lemon yellow; 3 ft. $\quad .50$

7776 Sherwin Wright (Koh) Golden yellow .25

8382 Silver Ribbon (Wil) Pink toned; $2 \frac{1}{2}$ ft. 1.50

8584 Simonne Vassieri (Mil) Lord of June type 1.50

8780 Sindjkha (Stur) Smoky lavender; $4 \mathrm{ft}$. . $\quad .35$

8584 Sir Galahad (Shull) Fragrant ........ 3.00

New Snow Bound (Rowe) Brown and white. 1.00

8587 Snow White (Stur) White; good size; 3 ft. 5.00 


\section{C. F. WASSENBERG, VAN WERT, OHIO}

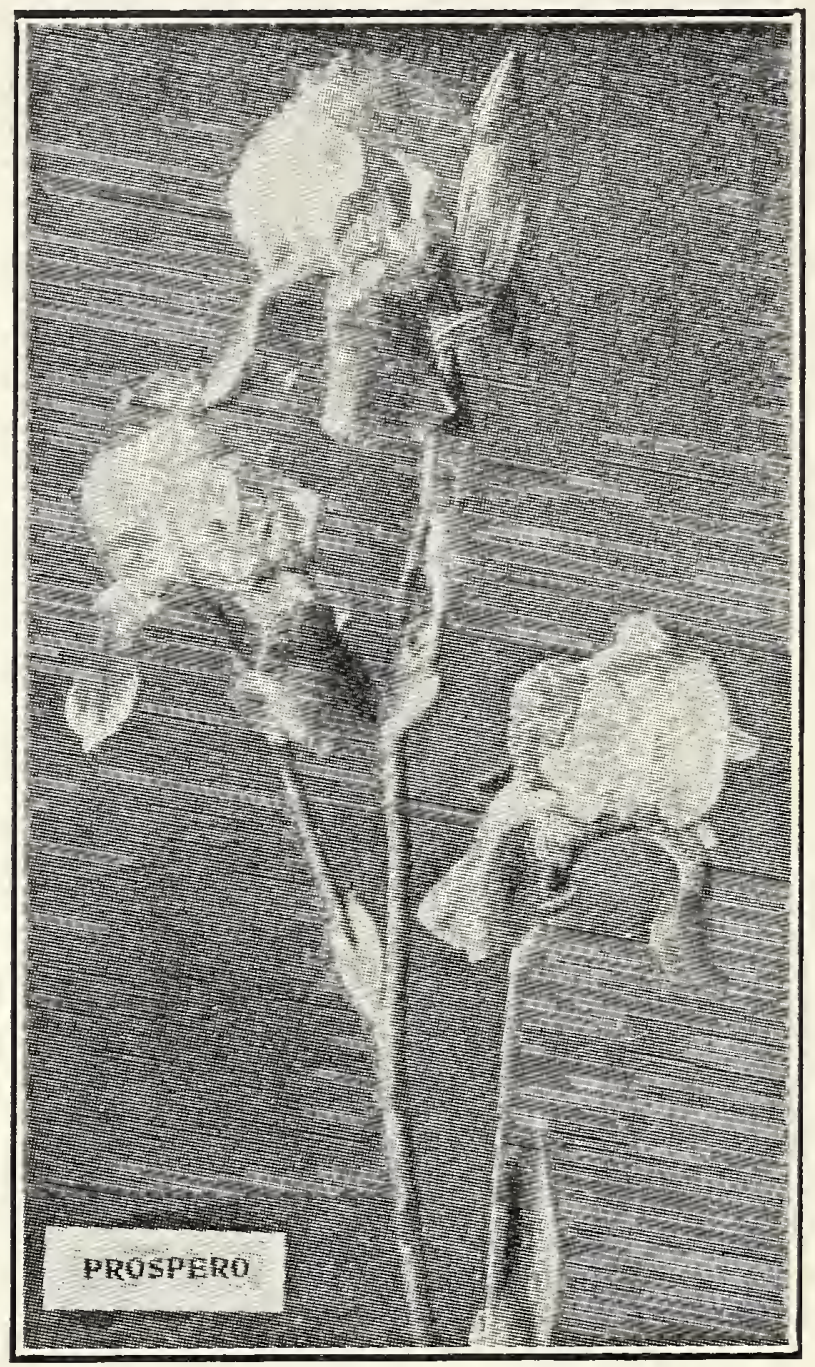

IRIS-PROSPERO

Large flower, beautiful color and good strong grower

9190 Souv. de Le Michaud(Mil)S.\&F. blue vio 3.50 9188 Souv. de Mme. Gaudichau (Mil) Dk pur. .75 7475 Syphax (Bliss) S. violet; F. crimson .. .25

7877 Stamboul (Fos) S. blue; F. lav. blue .... .50 8282 Steepway (Scott) Mauve; faun \& blue .75 8382 Sunset (Den) Variegated yel; late; good .75 8586 Susan Bliss (Bliss) Fine lilac pink .... 1.00 8282 Suzanne Autissier (Den) Blue violet pur. .75 8984 Swazii (Bliss) S. vio; F. dark pansy vio. 5.00 8587 Sweet Lavender (Bliss) French grey .... .75 8281 Taj Mahal (Stur) White; one of the best 1.50 72 Tamar (Bliss) Reddish lilac ......... .25 8888 Teneabrae (Bliss) S. vio.; F. violet purple 2.00 7979 Thecla (Wil) S. bright mauve; F. purple 1.00 New Thorsten (G\&K) Fragrant; blue bicolor 2.50 8482 Titan (Bliss) S. vio. blue; F. purple... 2.00 6783 Toreador (Clev) S. orange; F. red .... .50

\section{GET YOUR ORDER IN EARLY}


each

7374 Trianon (Vil) Pale buff; Free flowering .25

8585 Tropic Seas (Shull) S. vio.; F. blue pirpple 2.00

79 Troost (Den) Rose colored pink e îtect .50

8884 True Charm (Stur) White; edges blue lav. 150

8585 True Delight (Stur) Wht; rose markings 250

6966 Tunisie (Mil) Smoky heliotrope ....... .25

7275 Turco (Vil) Buff and violet ........... .25

Twilight (Sass) Soft blue ..... . 1.50

Tyrian (Stur) Vivid deep claret; $3 \mathrm{ft} .2 .53$

83 Ute Chief (Farr) S. violet; F. pur.; 3 ft. .35

8586 Valencia (Mohr) Orange buff salf; good 200

7982 Valery Mayet (Den) S. Rose; F red brown .50

8681 Valkyrie (Stur) Lake and maroon purple 2.50

New Van Cleve (Van) Dark purpie; tall 5.00

New Van Dyck (Vil) New; rare; early; 28 in. 3.50

New Vega (Vil) Yellow bicolor; rare ...... 5.00

8989 Vesper Gold (Wil) Yellow tones; tall . 3.50

8385 Vingolf (G\&K) S. creamy bronze; F. vio. 1.00

7376 Violacea Grandiflora (Wild) Violet blue . $\quad .25$

7677 Virginia Moore (Shull) Yellow: late .50

7779 Warrior (Stur) Rich purple; large; 3 ft. 1.50

New Watteau (Vil) Medium blue purple . 5.00

W. C. Terry (Per) S. violet; F. crim. pur. 1.50

8787 Wedgewood (Dyk Blue seif; well named 3.00

7980 White Knight (Saun) White; good; 2 ft. .50

8181 White Queen (Cay) Pure white; fine; .5)

7479 W. J. Fryer (Fry) S. yellow; F. mar. 3 ft. .35

7472 Wyomissing Creamy rose self .25

8585 Yellow Moon (Stur) Soft uniform yellow 1.50

8385 Yoeman (Bliss) S. light vio. F. deep vio. $2.0 \mathrm{~J}$

8782 Yolande (Mil) S. \& F. Dark blue purple 2.50

7981 Yvonne Pelletier (Mil) Pale blue; tall . .35

7881 Zada (Emig) A pure white; 3 ft. . . 2.00

7474 Zilia (Per) S. \& F. Pansy blue; tall; early 1.00

7576 Zouave (Vil) White and violet dotted .35

75 Zua (Craw) Wht, frosted crepe; crinkled .50

8585 Zulu (Bliss) S. vio.; F. blackish rich vio. 6.00 Zwanenburg Cream and maroon ...... .50

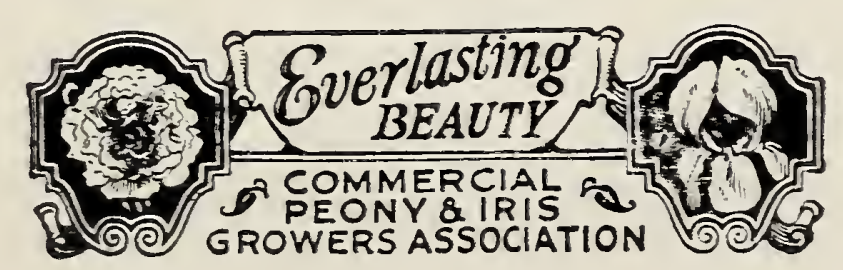

ALL RETAIL SHIPMENTS PREPAID 

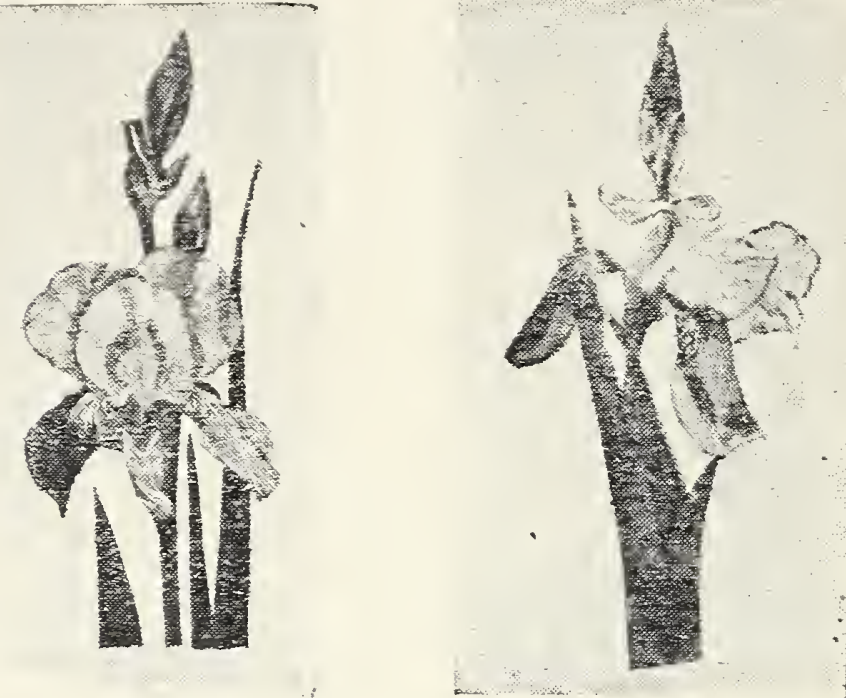

BEARDLESS IRIS

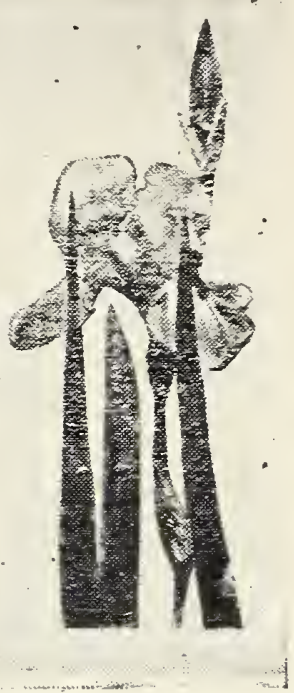

Each

Dorthea K. Williamson (Willm) Violet $\$ 1.00$

Orientalis, Blue King Brilliant blue _... $\quad .50$

Orientalis, Snow Queen Ivory white _... .50

Sibirica, Distinction $\quad .50$

Sibirica, Emperor Dark blue …..... .75

3ivirica, Grandis Dark blue _........ .50

Sibirica, Lactea White _........ .50

Sibirica, Mme. Butterfly (Cle) Clear blue .75

Sibirica, Perry's Blue (Per) Sky blue _ .75

Sibirica, Skylark Light blue ......... $\quad .75$

Sibirica, Sunnybrook (Cleve) Alice blue .75

Sibirica, True Blue (Fry) True blue - .50

Versicolor _...... .50

Pseudacorus Bright yellow _..__...... .50

DWARF BEARDED IRIS

Cyanea Red purple

Graminae Red violet _............. 50

Marican ..................... .50

Niobe Royal purple _.................. .35

Orange Queen Yellow .................. .50

Standard Light purple _.............. .50

The Bride White ................ .35

\section{JAPANESE IRISES-Assorted}

Single plants

each

$\$ 0.50$

Field clumps

1.00

ORIENTAL POPPIES

Beauty of Livermore Bright crimson

Lula A. Neeley Rich cardinal

Mrs. Perry Salmon pink

Wurtembergia Finest red; large 


\section{EXTRAORDINARY PEONY AND IRIS COLLECTIONS}

\section{IRIS COLLECTION NO. 1.}

9.1 Ambassadeur, S. bronze; F. mar. $\$ .50$

8.8 B. Y. Morrison. S. violet; F. purple .50

8.5 Dream, Light pink .......... . .50

Imperator, Improved Seminole . . . 1.00

9.6 L.A.Williamson, $S$ violet; $F$ purple .50

Mother of Pearl, Pearl tints ..... .50

8.7 Opera, Lilac and purple ....... .50

Prospero, Lavender and purple... 1.00

8.8 Shekinah, Lemon yellow ....... .50

8.3 White Knight, White ......... .50

PEONY COLLECTION NO. 1.

P.P.

Prepaid $\$ 4.00$

A fine assortment for beginners. Assorted shades and colors.

Edulis Superba. Early pink

Elwood Pleas. Light rose pink _._. 2.50

Felix Crousse, Bright red _... 1.00

Festiva Maxima. Pure white

La Perle. Soft pink

Mme. Ducel. Pink; bomb

P.P.

Prepaid

$\$ 5.00$

\section{PEONY COLLECTION NO. 2.}

$\$ 7.50$

Notice these seven most beautiful varieties and how little they cost.

Baroness Schroeder. Late white

Elwood Pleas. Light rose pink $\$ 1.50$

Jubilee. Prize white

Karl Rosefield. Dark crimson

Mons. Jules Elie. Large; deep pink

Solange. Finest dark cream

Therese. Soft pink; the best

1.50

3.50

3.00

$\$ 16.00$

P.P.

Prepaid

$\$ 10.00$

\section{PEONY COLLECTION NO. 3.}

These six you will be most proud to have in your garden.

Kelway's Glorious. Finest white . . .\$10.00 Longfellow. Cherry red .......... 3.00

Lady Alex. Duff. Pale pink; beautiful. 2.50

Milton Hill. Lilac rose; matchless ... 3.50

Mrs. E. Harding. White; exquisite . . . 10.00

Tourangelle. Flesh tinted salmon.... 3.00

Walter Faxon. Salmon rose 
MEMORANDUM 

PRINTED BY

THE TINGLE PRINTING CO. PITTSVILLE, MD. 